\title{
Pulsed Electrically Detected Magnetic Resonance for Thin Film Silicon and Organic Solar Cells
}

\author{
Alexander Schnegg, ${ }^{* a}$ Jan Behrends, ${ }^{a, b}$ Matthias Fehr ${ }^{a}$ and Klaus Lips***a \\ Received (in $X X X, X X X)$ th $X X X X X X X X X 20 X X$, Accepted $X$ th $X X X X X X X X X 20 X X$ \\ ${ }_{5}$ DOI: 10.1039/b000000x
}

In thin film solar cells based on non-crystalline thin film silicon or organic semiconductors structural disorder leads to localized states that induce device limiting charge recombination and trapping. Both processes frequently involve paramagnetic states and become spindependent. In the present perspectives article we report on advanced pulsed electrically detected magnetic resonance (pEDMR) experiments for the study of spin dependent transport processes in fully processed thin film solar cells. We reflect on recent advances in ${ }_{10}$ PEDMR spectroscopy and demonstrate its capabilities on two different state of the art thin film solar cell concepts based on microcrystalline silicon and organic MEH-PPV:PCBM blends, recently studied at HZB. Benefiting from the increased capabilities of novel pEDMR detection schemes we were able to ascertain spin-dependent transport processes and microscopically identify paramagnetic states and their role in the charge collection mechanism of solar cells.

\section{1. Introduction}

World energy demand is expected to more than double in the next 50 years. Conventional energy sources will not be adequate to supply this demand, without causing severe environmental and climate damage. This calls for the rapid development of abundant 20 sustainable energy sources. By far the largest carbon-neutral energy source is provided by the sun. The amount of solar power that strikes the earth in one year $\left(>100 \times 10^{15} \mathrm{~J}\right)$ clearly exceeds the annual global energy consumption $\left(15 \times 10^{12} \mathrm{~J}\right){ }^{1}$

The practicality of photovoltaic (PV) devices has been ${ }_{25}$ demonstrated by the technological development and successful commercialization of crystalline silicon (c-Si) solar cells, which created a rapidly growing billion euro market. By the end of 2011 more than $68 \mathrm{GW}$ of PV capacity has been installed worldwide, yet, PV energy provided less than $0.1 \%$ of world's electricity 30 needs. ${ }^{2}$ The reason for the huge mismatch between our present day PV use and its enormous undeveloped potential results from the fact that PV electricity production despite its impressive conversion efficiency ${ }^{3}$ and flexibility is still not fully price competitive to conventional energy sources.

${ }_{35}$ To bridge this gap, research aims at higher PV conversion efficiency and at the same time a reduction of material costs. Here, thin-film solar cells have the largest potential since they can be deposited on a large variety of inexpensive substrates with tailored physical and optical properties with significantly reduced 40 material consumption, which results in lower energy payback time and, in principle, also in better cost effectiveness. The main absorber materials used for thin-film solar cells are based on (i) hydrogenated amorphous and microcrystalline silicon (a-Si:H and $\mu \mathrm{c}-\mathrm{Si}: \mathrm{H}$, respectively) summarized as thin-film silicon (TFS) in 45 the following,, 5 (ii) cadmium telluride (CdTe), (iii) Copper indium (gallium) diselenide (CI(G)S), ${ }^{6}$ and (iv) dye sensitized ${ }^{7}$ and organic, molecular or polymeric organic photovoltaic (OPV) blends. ${ }^{8}$ Among the different materials TFS was the first to reach the stage of large-scale solar cell production, while OPV devices 50 just enter the stage of commercialisation. However, recent years witnessed the steepest increase of record efficiencies that have been demonstrated on the lab scale for organic blends. ${ }^{3,9}$ It is beyond the scope of the present perspectives article to discuss and compare different solar cell concepts. Instead, we want to focus 55 on performance limiting effects which involve paramagnetic states in TFS and OPV solar cells and which can be monitored through the detection of spin-dependent charge carrier transport and recombination. The major drawback of OPV and TFS solar cells are solar conversion performances being far below the ${ }_{60}$ physical limits determined by thermodynamics, the so-called Shockeley-Queisser limit. ${ }^{10}$ In TFS and OPV solar cells the PV efficiency limits are induced by material disorder which strongly reduces the open circuit voltage and fill factor. ${ }^{11}$ The most basic functional solar-cell unit is the light-generated excitation in the 65 absorber material (exciton). In a properly designed solar cell this exciton will lead with an efficiency of nearly $100 \%$ to charge separated states which are essentially unpaired (paramagnetic) electrons and holes. Optimum solar cell operation requires their non-dissipative extraction. Processes that trap or scatter charge 70 carriers can reduce the charge-carrier mobility or induce chargecarrier recombination and thereby decrement solar cell efficiencies. Consequently, detailed insight into trapping and recombination processes may help to develop advanced material engineering strategies for improved solar cell performance. It is 75 therefore of major importance to understand the genesis of such trap states and their role in the charge collection mechanism.

Due to the paramagnetic nature of the charge carriers, recombination and trapping mechanisms in thin-film solar cells are frequently accompanied by stable or transient paramagnetic

80 states, where the spins of light-generated charge carriers interact with each other or with other paramagnetic sites in the material. These electron and sometimes also nuclear spins constitute 
protruding probes to study the impact of device limiting defects and impurities at interfaces and in the bulk.

During a trapping, tunnelling or recombination event between two paramagnetic states in a given solar cell material, the electron 5 spins will be in close proximity of each other and, depending on their transition probability, will form coupled spin pairs with a certain spin coherence lifetime. If the formation or dissociation of these spin pairs changes the conductivity of the sample, electric transport will become spin-dependent.

10 By measuring the coupling energies, life and correlation times of these spin pairs the transport determining site can be identified and directly ascertained to the underlying charge transport or loss mechanism. The method of choice to study the dynamics and the electron spin-coupling energies is provided by electron 15 paramagnetic resonance (EPR).

However, the sensitivity of microwave (mw) detection techniques ( $10^{10}-10^{13}$ spins) is usually not sufficient to monitor EPR in state of the art thin-film solar cells with active layer thicknesses in the $\mu \mathrm{m}$ to $\mathrm{nm}$ range. Moreover, the interpretation of EPR 20 signals from fully processed thin-film solar cells may be hampered by EPR signals from the substrate, which have no relevance for the solar cell operation. ${ }^{12}$

In solar cells the sensitivity and selectivity limit of EPR can be lifted by electrically detected magnetic resonance (EDMR). ${ }^{13-15}$

${ }_{25}$ EPR and EDMR are similar in that both monitor the resonance fields of mw-induced electron spin-flips. Their difference consists in the detection scheme. EDMR monitors spin-dependent current changes instead of mw absorption (a more detailed description of EDMR detection is given in Section 3). Electrical detection 30 makes EDMR selective to paramagnetic transitions, which impact charge transport processes while paramagnetic background signals are inherently suppressed. As it combines the nanoscopic information content of conventional EPR with the sensitivity of a current measurement, EDMR provides a tool to directly study the 35 influence of paramagnetic states on the dark or photocurrent with a detection sensitivity approaching single spin detection. ${ }^{16-18}$

The particular use of EDMR in solar cell research arises from its capability to identify device limiting loss mechanisms by determining structural information of transport limiting 40 paramagnetic sites like e.g. impurities or defects. ${ }^{19-24}$ A profound understanding of this structure-function relationship is mandatory to rationalize and optimize the material properties and the manufacturing process of thin film solar cell devices.

Recently it was shown that the information content extractable 45 from EDMR measurements can be even increased by combining electrical detection with pulsed mw excitation schemes. ${ }^{25,} 26$ Following the pathway of advanced pulsed $\mathrm{EPR}^{27}$ the successful implementation of pEDMR paved the way for novel detection schemes, ${ }^{26,28-37}$ which greatly increased the spectral resolution as 50 well as the selectivity to different spin-dependent transport mechanisms and spin coupling parameters.

From the onset the development of pEDMR was driven by applications to thin-film Si materials ${ }^{29,} 38-46$ and solar cells. ${ }^{47-50}$ More recently the approach has been extended to their organic 55 counterparts. ${ }^{51-55}$

In the present perspectives article we outline the function of pEDMR and how it may be employed to assign and characterize spin-dependent processes in fully processed TFS and OPV devices. In particular, we will show that despite their dissimilar 60 material composition general conclusions may be drawn on spindependent transport mechanisms in organic and disordered silicon materials.

The manuscript is organized as follows: Section 2 introduces the theoretical foundations of spin-dependent transport processes in ${ }_{65}$ TFS and OPV materials. Special emphasis is put on spindependent hopping and recombination as dominating processes. Section 3 outlines the basic principles and technical requirements of pEDMR detection. pEDMR studies in the working principles of a-Si:H/ $\mathrm{Lc}-\mathrm{Si}: \mathrm{H}$ pin and polymer MEH-PPV/PCBM solar cells 70 are presented in Section 4 and 5, respectively. The main results of this article are summarized in section 6 and the implications and perspectives of advanced pEDMR methods on future solar cell developments and application of pEDMR beyond this field are highlighted in the outlook Section 7.

\section{$75 \quad 2$ Spin-dependent transport}

In the following we will outline under which conditions transport-related paramagnetic states may be encountered in TFS and OPV materials, alongside with an introduction of the dominating transport processes involving these states.

${ }_{80}$ In TFS devices charge transport is governed through extended states (band transport). The spins of delocalized electrons and holes in the conduction and valence bands strongly interact with each other and the host lattice, so that the spin orientation of one specific charge carrier is not conserved for long times, except at 85 very low temperature and/or high magnetic fields, accompanied by a significant spin polarization. This situation is dramatically different for charge carriers trapped in localized defect states in the band gap of a semiconductor (e.g. three fold coordinated Si atoms, so called dangling bonds (db)) or band-tail states. These 90 sites are often paramagnetic or can be made paramagnetic through electronically or structurally induced processes (e.g. charge injection or doping induced charge transfer). Paramagnetic defects impact the performance of TFS solar cells via recombination or alternation of the charge carrier mobility.

95 In the case of organic semiconductors, hopping of charge carriers via localized states (positive $\left(\mathrm{P}^{+}\right)$and negative polarons $\left(\mathrm{P}^{-}\right)$) is the dominating charge transport mechanism. In addition, the weak spin-orbit coupling in organic materials results in relatively long spin-relaxation times. In this case the charge transport 100 process involves long-lived transient paramagnetic states. This results in the fact that spin states even at room temperature may have dramatic influence on charge transport and recombination.

However, both material systems share general mechanisms that render charge transport spin-dependent. Whenever a tunnelling 105 process from one localized state to another occurs between two paramagnetic states, spin selection rules may govern the transition probability, provided that the spin is conserved throughout the transition. In this case the tunnelling rate may depend on the relative spin orientation of both charge carriers. 110 Through this spin coupling effect charge carriers form spin pairs prior to the transition process. A static magnetic field will affect the energy of such spin pairs (e.g. through the Zeeman splitting) and may thus alter the tunnelling rate through various processes. Irrespective of the underlying microscopic mechanisms, this 115 translates into a conductivity change observed in 
magnetoresistance measurements in which the resistance (or conductivity) of a sample is measured upon changing the amplitude of the static magnetic field. Magnetoresistance in disordered systems such as a-Si:H was observed already several 5 decades ago. ${ }^{56,57,58}$ While the resulting magnetic field induced change in conductivity was typically restricted to less than one percent in a-Si, this effect recently generated renewed interest when large magnetoresistance exceeding $10 \%$ was observed in organic semiconductors at room temperature. ${ }^{59}$ This illustrates 10 that, particularly in organic semiconductors, the spin has a dramatic influence on charge transport and thus also affects the carrier collection efficiency in solar cells made from this material. Thus, it is important to understand the underlying mechanisms on a microscopic level. Based on magnetoresistance measurements 15 only this is complicated because such a measurement solely detects changes of a macroscopic observable, i.e., the conductivity, but does not provide microscopic insight. As a result, the origin of the magnetoresistance effect remains a topic of controversy, although systematic measurements were 20 performed using various experimental conditions and device configurations. $^{52,60,61}$

In order to complement the information obtainable from magnetoresistance measurements, it is helpful to actively manipulate the tunnelling rates that give rise to the change in 25 resistivity. This can be done by EDMR. In essence, an EDMR experiment can be regarded as a magnetoresistance measurement with the added possibility to actively manipulate the tunnelling rates between specific spin pairs responsible for the magnetoresistance effects by EPR. Through the spin flip the spin-

30 pair configuration will change which can lead to a change of the transition rate between the two spin-pair partners that can be observed as a change of the sample conductivity. Thereby the extractable information may be dramatically increased as compared to magnetoresistance experiments. Resonant spin 35 manipulation through EPR does not only provide information on spin-dependent transport mechanisms, but also on the local structure of paramagnetic states involved in respective transport processes. This selectivity to particular transport processes in combination with structural information on the nanoscale is a 40 unique feature of EDMR based techniques.

In order to attain this information the sample has to be exposed to a static magnetic field $\boldsymbol{B}_{\mathbf{0}}$. As a consequence the electron spins align parallel (spin up, $m_{S}=1 / 2$ ) and anti parallel (spin down, $m_{S}$ $=-1 / 2$ ) to $\boldsymbol{B}_{\mathbf{0}}$, which leads to the Zeeman energy difference $\Delta E$.

${ }_{45}$ Further modification of $\Delta E$ results from coupling of the electron spin to nearby electron and nuclear spins. Prerequisite of many spin-dependent transport processes are coupled spin pairs. In the presence of an external magnetic field the spin coupling energies of two coupled electron spins $\mathrm{A}$ and B $\left(S_{\mathrm{A}}=1 / 2\right.$ and $\left.S_{\mathrm{B}}=1 / 2\right)$ 50 may be most conveniently expressed by the following Spin Hamiltonian (SH). ${ }^{62}$

$$
\begin{aligned}
& H_{0}=\mu_{\mathrm{B}} \boldsymbol{S}_{\mathrm{A}} \cdot \boldsymbol{g}_{\mathrm{A}} \cdot \boldsymbol{B}_{0}+\mu_{\mathrm{B}} \boldsymbol{S}_{\mathrm{B}} \cdot \boldsymbol{g}_{\mathrm{B}} \cdot \boldsymbol{B}_{0} \\
& -J \hbar\left(S^{2}-1\right) \quad-S_{\mathrm{A}} \cdot \boldsymbol{D}_{\text {dip }} \cdot \boldsymbol{S}_{\mathrm{B}}+\sum_{i=\mathrm{A}, \mathrm{B}} \sum_{j} \boldsymbol{I}_{i j} \cdot \boldsymbol{A}_{i j} \cdot \boldsymbol{S}_{i}
\end{aligned}
$$

In Eq. 1, the first two terms denote the electron Zeeman ${ }_{55}$ interaction, which couples the electron spins $\boldsymbol{S}_{\mathrm{A}}$ and $\boldsymbol{S}_{\mathrm{B}}$ to $\boldsymbol{B}_{\mathbf{0}}$ via the anisotropic g-tensors, $\boldsymbol{g}_{\mathrm{A}}$ and $\boldsymbol{g}_{\mathrm{B}}$, respectively. The third and the fourth term are exchange and dipolar interaction terms of electron spin $\mathrm{A}$ and $\mathrm{B}$. These interactions are determined by electron-electron exchange ( $J$, with $\boldsymbol{S}=\boldsymbol{S}_{\mathrm{A}}+\boldsymbol{S}_{\mathrm{B}}$ ) and dipolar $\left(\boldsymbol{D}_{\text {dip }}\right)$ 60 coupling constants. In the case of weak electron-electron coupling the energy eigenstates are the product states $|1\rangle=|\uparrow \uparrow\rangle$, $|4\rangle=|\downarrow \downarrow\rangle,|2\rangle,|3\rangle$. The former two states are pure triplet (T) states. In contrast, the last two states possess singlet (S) and triplet character. ${ }^{63}$ The last term in Eq. 1 represents the hyperfine 65 interaction (HFI), which quantifies the coupling of the $i$-th electron spin with the $j$-th nuclear spin $\left(I_{j}\right)$, by the anisotropic HFI-tensor, $\boldsymbol{A}_{i j}$. The spin coupling parameters $\boldsymbol{g}, \boldsymbol{A}, J$ and $\boldsymbol{D}_{\text {dip }}$ are the target of magnetic resonance studies as they contain unique information on the electronic and local structure of paramagnetic 70 states. $^{27,62,64}$ Electron spins exposed to a static magnetic field may be flipped by applying an additional oscillating magnetic field $B_{1}$ with a frequency $v=\Delta E / h$. This is the EPR condition.

In EDMR the observable is the conductivity,

$\sigma=e\left[n_{\mathrm{e}} \mu_{\mathrm{e}}+n_{\mathrm{h}} \mu_{\mathrm{h}}\right]$

75 This is the major difference to EPR where the observable is the mw absorption. In Eq. $2 e$ is the elementary charge and $n_{\mathrm{e}}$ and $n_{\mathrm{h}}$ denote the concentrations of electrons and holes with mobilities $\mu_{\mathrm{e}}$ and $\mu_{\mathrm{h}}$, respectively. EPR induced changes in the conductivity $(\Delta \sigma)$ directly translate into changes in the electrical current $(\Delta I)$ 80 measured by EDMR. It is obvious from Eq. 2 that these changes may either result from a variation in charge carrier concentrations or mobilities. However, understanding under which conditions a spin flip changes the sample conductivity requires some more considerations.

85 The most important spin-dependent processes in TFS and OPV materials are spin-dependent charge-carrier recombination ${ }^{65}$ and spin-dependent hopping-transport. ${ }^{66}$ Both mechanisms are encountered in thin Si films ${ }^{67-69}$ and devices ${ }^{67,70}$ as well as their organic analogues. ${ }^{71,72}$ In the following we will outline the basic 90 principles of these processes, and relate them to the EDMR observable. In principle EDMR processes may result from other processes like e.g. spin-dependent charge carrier scattering, ${ }^{20,} 73$ spin-dependent charge carrier trapping and reemission ${ }^{74,75}$ or bolometric effects. ${ }^{76}$ All above mentioned spin-dependent process 95 can lead to very complex modulation of the charge carrier density and hence contribute in very different ways to the EDMR signal. In case non-ohmic contacts are used, spin-dependent trap-assisted tunnelling through contact barriers can also play a role. ${ }^{77}$ It requires a very careful analysis of the spin-dependent process, 100 and in many cases device simulation is an essential requirement to distinguish between different processes. ${ }^{12,} 78$ In the following we will focus on only those spin effects which are believed to dominate the EDMR of the solar cell devices, which were studied in the present work. 


\section{spin-dependent recombination}

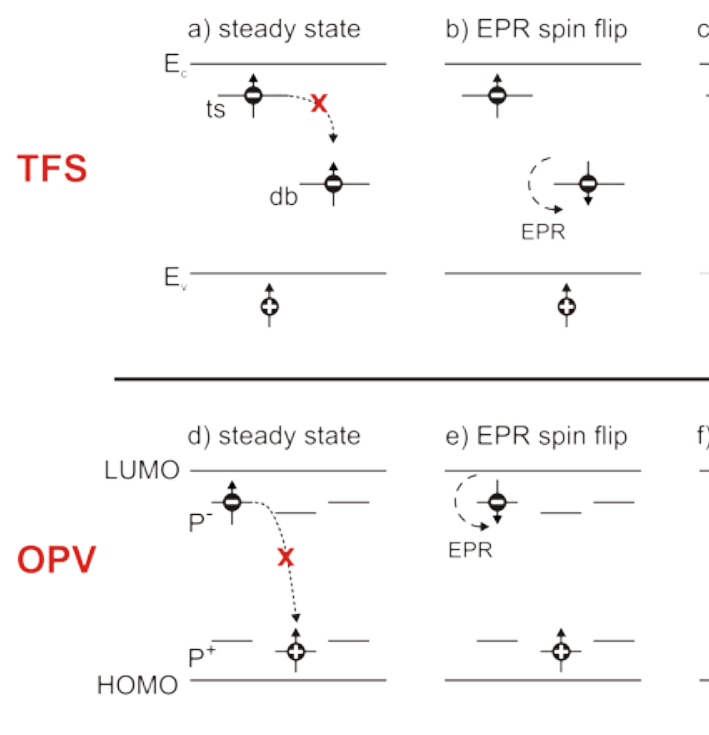

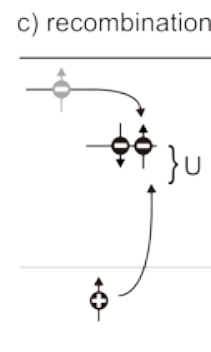

spin-dependent hopping
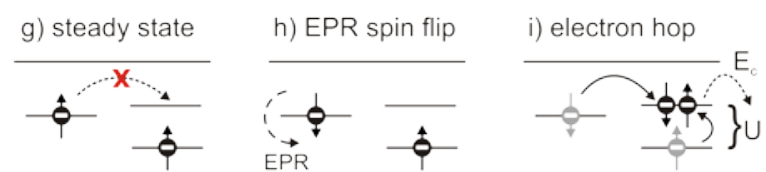

Fig. 1 Spin-dependent (left) charge carrier recombination and (right) hopping-transport involving (a) to c) and g) to i)) localized states below the conduction $\left(E_{\mathrm{C}}\right)$ and above the valence bands $\left(E_{\mathrm{V}}\right)$ of TFS and (d) to f) and j) to l)) the polaron levels ( $\mathrm{P}^{+}$and $\left.\mathrm{P}^{-}\right)$between the lowest unoccupied orbital 5 (LUMO) and the highest occupied orbital (HOMO) of OPV materials. + and - indicate positive and negative charges, while the spin orientation is shown by arrows pointing up or down. $U$ denotes the correlation energy.

\section{Recombination:}

a) In TFS excess electrons may be captured by tail states (ts) localized below $E_{\mathrm{C}}$ and above $E_{\mathrm{V}}$, respectively. These electrons can tunnel further into energetically lower lying dangling bond $(\mathrm{db})$ states. If the target energy level is already singly occupied before the transition, this process is only allowed

10 if the respective spins are in singlet state. In the depicted case of parallel spin alignment recombination is blocked. b) The relative spin orientation may be flipped by resonant EPR excitation of either of the spins. c) Once the dangling bond state is doubly occupied, a hole from $E_{\mathrm{V}}$ or from a valence band-tail state (not shown) can be captured in the db state to accomplish the recombination step. d) In OPV materials charge transport is governed by polarons. A $\mathrm{P}^{-}$ may encounter a trapped $\mathrm{P}^{+}$and form a coupled spin pair. Similar to the TFS case the polarons cannot recombine if their unpaired electron spins form a triplet state. e) EPR spin-flips lead to f) direct recombination between $\mathrm{P}^{+}$and $\mathrm{P}^{-}$.

15 Hopping:

g) Charge carriers in TFS (only transport of electrons shown here) that relax to tail states may perform hopping steps between adjacent localized states. These are spin-dependent when the target energy level is singly occupied before the hop, and the hopping transition produces a doubly occupied site. h) Blocking of the transport path by spin pairs in triplet state may be lifted by EPR. i) After flipping one of the coupled spins the electron can hop onto the singly occupied state and propagate further. j) In OPV materials $\mathrm{P}^{+}$and $\mathrm{P}^{-}$polarons migrate through the material (only $\mathrm{P}^{+}$transport shown here). Again,

20 the probability for a hop of one polaron to a site that is already singly occupied depends on the relative spin orientation of both polaron spins. k) Flipping one of the spins l) lifts again the transport blockade. In TFS and OPV materials hopping may involve positive and negative charges.

\section{1) Charge carrier recombination}

Common to charge carrier recombination and hopping-transport 25 is that both can be described in the framework of the spin-pair model developed by Kaplan, Solomon and Mott which is referred to as the KSM model. ${ }^{79}$ Fig. 1 depicts the impact of spin-pair formation on spin-dependent recombination (left) and hopping (right) in TFS (top) as well as OPV (right) materials.

${ }_{30}$ Fig. 1 a)-c) shows recombination via a paramagnetic impurity (e.g. a db) located energetically in the band gap of the semiconductor material. This case is frequently encountered in TFS materials. Prior to the recombination event spin pairs are formed by negative charges captured in tail states and 35 energetically lower lying singly occupied (e.g. db) defects.
Although it would be energetically favourable if both carriers occupied the lower-lying state, owing to the Pauli-principle this transition is only allowed when both electron spins are aligned anti parallel. Thus, invoking that the spin state is conserved 40 during the transition process, the transition is blocked in the case of parallel spins. The recombination rate coefficient associated with the triplet states is $r_{\mathrm{T}}$, i.e. the triplet recombination rate coefficient. For singlet states the recombination rate coefficient is given by $\left(r_{\mathrm{S}}+r_{\mathrm{T}}\right) / 2$, for the case of negligible coupling. For any 45 spin-pair state the recombination rate coefficient can be expressed by

$$
r_{i}=r_{\mathrm{S}}|\langle i \mid \mathrm{S}\rangle|^{2}+r_{\mathrm{T}}\left|\left\langle i \mid \mathrm{T}_{0}\right\rangle\right|^{2},
$$


i.e. is determined by the singlet and triplet content of the spinpair.

The application of mw radiation resonant with either of the two spin-pair constituents leads to an EPR spin-flip (Fig. 1 b)). The 5 initially forbidden transition becomes allowed. In essence, the singlet content of a given pair increases, resulting in an enhanced recombination probability of the spin pair. The recombination process is then completed by the capture of a hole from the valence band (Fig. $1 \mathrm{c}$ )). For a more detailed description of the 10 underlying spin physics see e.g. Ref. ${ }^{80}$ In OPV materials spindependent charge carrier recombination typically occurs between $\mathrm{P}^{+}$and $\mathrm{P}^{-}$and hence directly affects the current-carrying species without the necessity of trapping additional charge carriers at the recombination site (Fig. $1 \mathrm{~d}$ )) to f)). Although details of the 15 recombination process are different for inorganic and organic solar cells, the influence of spin-dependent recombination on the conductivity can in both cases be described using the same set of equations. $^{28,33}$ In both material systems recombination annihilates one positive and one negative charge, resulting in a decrease of $n_{\mathrm{e}}$ 20 and $n_{\mathrm{h}}$ and thus, according to Eq. 2, a change in sample conductivity by

$$
\Delta \sigma=e\left[\Delta n_{\mathrm{e}} \mu_{\mathrm{e}}+\Delta n_{\mathrm{h}} \mu_{\mathrm{h}}\right]
$$

where $\Delta n_{\mathrm{e}}$ and $\Delta n_{\mathrm{h}}$ denote the EPR-induced deviations of the concentrations of negative and positive charge carriers from their

25 respective steady-state values. In inorganic semiconductors $n_{e}$ and $n_{h}$ usually represent the concentrations of delocalized charge carriers, i.e. electrons in the conduction band and holes in the valence band, whereas they refer to less mobile charge carriers $\left(\mathrm{P}^{+}\right.$and $\left.\mathrm{P}^{-}\right)$in most organic semiconductors.

\section{2.2) Hopping-transport}

Spin-dependent hopping-transport occurs when tunnelling between paramagnetic localized states dominates charge carrier transport. This applies to charge carrier collection in TFS solar cells, ${ }^{81}$ particularly at low temperatures, and in OPV cells ${ }^{82}$ which 35 is shown schematically in Fig. $1 \mathrm{~g}$ )-i) and $1 \mathrm{j}$ )-l), respectively. The spin-dependent transport step is the hop of a single charge carrier to a singly occupied state having similar energy. The hopping rate is generally determined by the localisation length and the separation as well as the energy difference between the ${ }_{40}$ two localized states. ${ }^{81}$ Note that the size of the correlation energy $U$ (the energy difference between singly and doubly occupied states) plays an important role here. For spin-dependent hopping to occur, $U$ must necessarily be smaller than the width of the distribution function for the energies of the localized states. ${ }^{83}, 84$

45 This is certainly the case for a-Si:H, where the band-tail states extend far into the gap, and the correlation energy is on the order of $10 \mathrm{meV}^{85}$ In organic semiconductors $U$ is much larger, typically several hundred meV. The width of the defect distribution varies for different materials, but is often found to be 50 in the same range. ${ }^{86}$

Analogous to the recombination process described before, the transition between adjacent singly occupied states can be blocked by the relative spin orientation (as shown in Fig. $1 \mathrm{~g}$ ) for TFS and j) for OPV materials). Again, EPR excitation (Fig. 1 h) and k)) 55 can flip one spin and enable an initially forbidden transition (Fig. 1 i) and 1)). The enhancement of the hopping rate can be considered to enhance the mobility (given by $\mu_{\mathrm{e}}$ or $\mu_{\mathrm{h}}$ ) in the hopping-transport path. In consequence, a conductivity change

$$
\Delta \sigma=e\left[n_{\mathrm{e}} \Delta \mu_{\mathrm{e}}+n_{\mathrm{h}} \Delta \mu_{\mathrm{h}}\right]
$$

60 results. Depending on the charge of the hopping carrier (electrons or holes in TFS and positive or negative polarons in organic semiconductors (OSC)) the hopping step affects either $\mu_{\mathrm{e}}$ or $\mu_{\mathrm{h}}$. Independent of the material system and the transport mechanism, spin-dependent transport processes based on the KSM model 65 share a number of important peculiarities.

A complete description of transient spin-dependent conductivitychanges following pulsed EPR excitation requires knowledge about the fate of the charge carriers before and after spin-flip. ${ }^{80}$

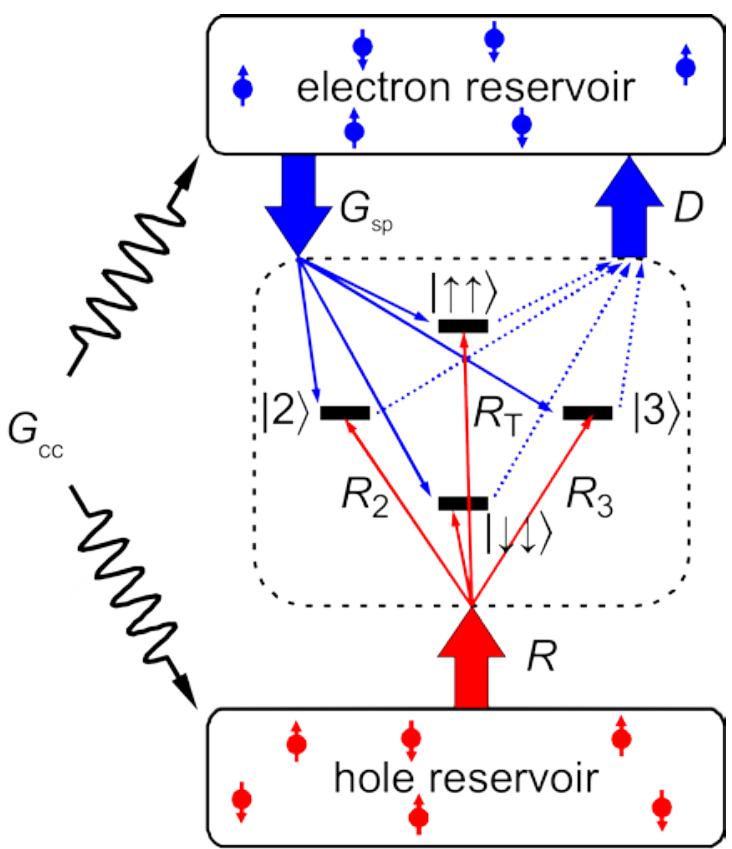

70 Fig. 2 Rate equations describing the dynamics of the spin pairs and the free charge-carrier concentrations. Free electrons and holes are created by light absorption or electrical injection (at the rate $G_{\mathrm{cc}}$ ) and act as reservoirs which are connected to the spin-pair ensemble via generation $\left(G_{\mathrm{sp}}\right)$, recombination $(R)$ and dissociation $(D)$.

75 The free (or mobile) charge carriers that determine the conductivity and localized spin pairs that determine the EDMR response are connected via spin-pair generation, dissociation and recombination. The interrelation between these quantities can be expressed by a set of coupled rate equations that are illustrated in 80 Fig. 2 (for details see Ref. ${ }^{87}$ ). Free charge carriers may be generated by electrical injection or light excitation at the rate $G_{\mathrm{CC}}$. These electrons and holes (TFS) or polarons (OPV) act as reservoir. For spin-dependent recombination they are connected to the spin-pair states via spin-pair generation $\left(G_{\mathrm{SP}}\right)$, dissociation ${ }_{85}(D)$ and recombination rates $(R) . R$ is spin-dependent whereas $G_{\mathrm{SP}}$ and $D$ are spin-independent. In addition, the influence of spinindependent recombination on $n_{\mathrm{e}}$ and $n_{\mathrm{h}}$ has to be taken into account, which is usually done by a charge carrier life time $\tau_{\text {. }}$. $G_{\mathrm{SP}}$ is proportional to $n_{\mathrm{e}}$. while $D$ results in an increase of $n_{\mathrm{e}}$. $R$ 90 directly reduces $n_{\mathrm{h}}$ and indirectly leads to a decrease of $n_{\mathrm{e}}$ due to reduced $D$. In the case of spin-dependent hopping spin pairs are 
also connected to the charge-carrier reservoir by a characteristic pair generation rate $\left(G_{\mathrm{SP}-\mathrm{H}}\right)$. Further, spin-pair dissociation may involve spin-dependent or spin-independent processes. Both possibilities have to be taken into account to quantitatively 5 describe hopping-induced mobility changes. A complete model of both spin-dependent recombination as well as hopping-transport further has to include spin relaxation processes. Spontaneous spin-flips occur within the spin-lattice relaxation time $T_{1}$; thereby the respective transport channel is switched by converting triplet 10 into singlet states. Numerical solutions of the resulting coupled rate equations may be found in Ref. ${ }^{28,}{ }^{80}$ Similar models have proven to be successful in modelling cwEDMR results in the past. ${ }^{88-92}$

A major challenge of EDMR is to link the relatively small 15 induced current changes, $\Delta I$, to the dominant transport channel in the materials of the device. Typically relative current changes $\Delta I / I$ are in the range from $10^{-7}$ to $10^{-2}$. Note that such small signal amplitudes are predicted even when the spin dependence is coupled to the dominating transport process in the device. The 20 absolute value of $\Delta I / I$ is determined by temperature, injection rate, illumination conditions, mw power, frequency and, most importantly, on the assumed transport process. A quantitative estimate of $\Delta I / I$ for different spin-dependent transport mechanisms in TFS may be found in Ref.. ${ }^{20}$ Relating EDMR 25 signals to overall transport properties in the working device is further complicated by the fact that, due to sensitivity reasons, most pEDMR studies are carried out at low temperatures. This usually requires complementary measurements and device simulations which should include the spin-dependent mechanism.

30 Depending on the details of the spin-coupling parameters of the involved spin-pair partners the EDMR signal shape and amplitude may change dramatically. ${ }^{93,} 94$ In the case of weakly coupled spin pairs EDMR spectra with two distinct resonance positions at their respective g values are predicted. Only one 35 single line is expected, even in cases where a spin-pair mechanism is evident when the resonance signals of both spin partners overlap due to similar spin-coupling parameters (see Eq. 1) or the interacting spins have dramatically different line widths. In this case sometimes only the narrow line may be detectable for 40 a given signal to noise level.

\section{Experimental foundations of pEDMR}

pEDMR provides a unique tool to study spin-dependent transport processes in semiconductor devices and in particular solar cells. However, pEDMR experiments require dedicated contact and 45 detection schemes. Despite the fact, that a growing number of research groups worldwide rely on this technique, $31,54,75,95-98$ pEDMR spectrometers are up to now commercially not available. In the following we outline the working principle of advanced pEDMR detection together with the pEDMR set-up and solar cell 50 sample designs developed in the EPR/EDMR lab at HZB. ${ }^{39,49,80}$

\section{1) pEDMR detection}

Fig. 3 a) depicts the basic pEDMR excitation scheme. ${ }^{25}$ The observable are current transients, $\Delta I$, following excitation by intense mw pulses. By integrating $\Delta|I|$ over time, a charge $Q$ is 55 obtained. Transient pEDMR spectra may be recorded by integrating $I(t)$ at different time intervals after the mw pulse and as a function of the external magnetic field $B_{0}$ under continuous charge generation by light or applied forward applied bias.
The evolution of a spin pair under EPR excitation is sketched in ${ }_{60}$ Fig. 3 b). Before the mw is switched on $(t=0)$, the spin pairs predominantly populate the long living triplet states. During the mw pulse the spin-pair states are manipulated and their population starts to coherently oscillate. After switching off the pulse, the spin-pair ensemble is no longer in the steady-state. 65 Therefore, spin-dependent recombination or hopping rates deviate from their respective steady-state values and affect the sample conductivity. While the spin manipulation typically takes place in the range from 10 to a few 100 ns, the detection of the resulting transient current change (referred to as pEDMR 70 transient) is performed for some $100 \mu$ s until the current has relaxed to its steady-state value $(\Delta I=0)$.

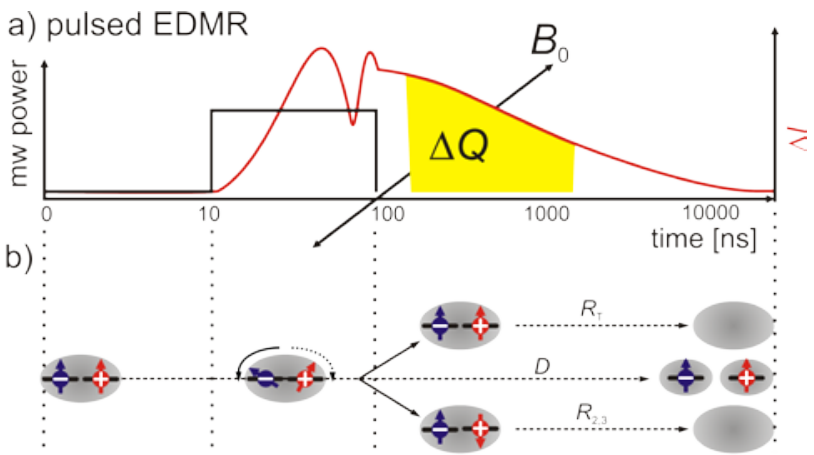

c) pEDMR detected Rabi oscillations

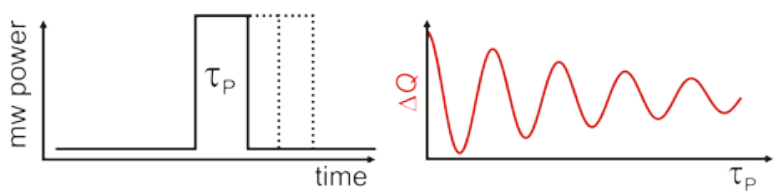

d) Echo detected pEDMR

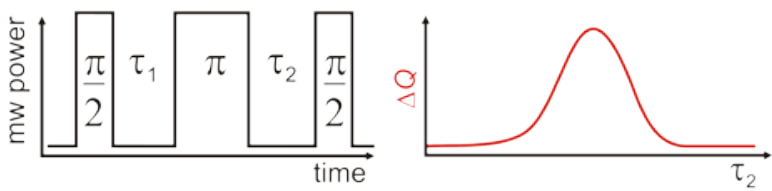

e) Electrically detected ESEEM

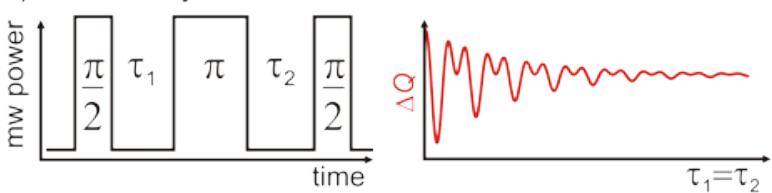

f) Electrically detected pulse ENDOR
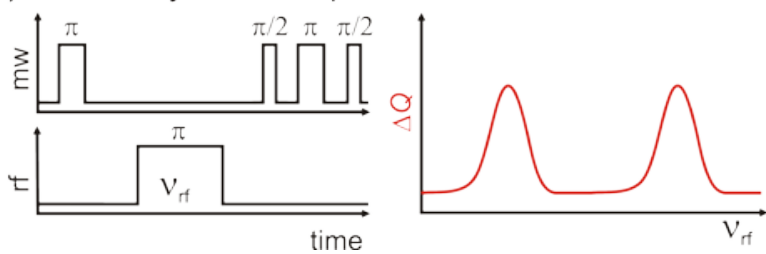

Fig. 3 pEDMR excitation and detection schemes a) Time-dependence of mw power (black) and spin-dependent current transients (red) recorded as a function of the external magnetic field $B_{0}$. (b) Spin-pair evolution during 75 the experiment. The pairs start from pure triplet states, are manipulated during the mw pulse and are annihilated by recombination (with rate coefficients $R_{\mathrm{T}}, R_{2}, R_{3}$ ), hopping or dissociation (with rate coefficient $D$ ) according to their singlet or triplet character. c)-f) Advanced pEDMR excitation schemes based on a variation of the mw and radio frequency 80 (rf) excitation pattern. 
Employing state of the art pulsed EPR instrumentation in combination with ultra-sensitive current detection, dedicated pEDMR excitation schemes have been designed to specifically address the electron spin couplings of paramagnetic states 5 involved in spin-dependent transport processes. In the following we will introduce the pEDMR detection schemes summarized in Fig. 3 c) to f) and highlight their particular use while studying spin-dependent transport processes.

To observe electron spin Rabi oscillations by means of pEDMR 10 (Fig. 3 c)), the charge $Q$ resulting from the integration of $\Delta|I|$ over several microseconds after the mw excitation is recorded as a function of the pulse length $\tau_{\mathrm{p} .}{ }^{28,80,99}$ Rabi oscillations allow for discrimination between spin-dependent transport processes that involve coupled paramagnetic centres with overlapping EDMR 15 spectra from those in which the spin-dependent transition takes place between two centres with spectrally separated EDMR resonances, i.e. different $g$ or A values..$^{35,41,53}$ In the latter case, one can address the spins of both sites separately by choosing the resonance condition according to the resonance position of the

20 respective site. The frequency of the coherent spin motion is then given by the Rabi frequency of the resonant paramagnetic centre provided that the driving field $\gamma B_{1}$ ( $B_{1}$ denotes the amplitude of the mw magnetic field and $\gamma$ is the gyromagnetic ratio) is small compared to the Larmor separation $\Delta \omega=\left(\omega_{\mathrm{A}}-\omega_{\mathrm{B}}\right)$ of the two 25 respective centres. ${ }^{93}$ Here, $\omega_{\mathrm{A}}$ and $\omega_{\mathrm{B}}$ are the Larmor frequencies of spin A and B (see Eq. 1), respectively. When the Larmor separation of two participating centres is smaller than the driving field, the Rabi frequency increases by a factor of two (spin locking case). ${ }^{100}$

30 The temporal damping of spin-Rabi oscillations is determined by the fate of the electron spin-pairs, in particular recombination, dissociation and hopping as well as by dephasing of the electron spins. These processes can be discriminated by echo detected pEDMR measurements. Electrically detected electron spin echoes 35 may be created by the modified Hahn-echo sequence depicted in Fig. 3 d). ${ }^{34,} 75$ Rotary echoes ${ }^{26,32}$ have been employed for pEDMR studies in electron spin dephasing and relaxation mechanisms as well as to investigate the process of spin-pair generation. In mw detected EPR spin echoes are formed by a $\pi / 2-$

${ }_{40} \tau_{1}-\pi-\tau_{2}$ - sequence. $\pi$ denotes the flipping angle of the electron spin, whereas $\tau_{1}$ and $\tau_{2}$ are the free evolution periods between the pulses. For electrical detection of the spin echo the standard two pulse sequence is extended with a $\pi / 2$ readout pulse at the time of echo formation. ${ }^{34}$ The final pulse transfers electron coherence to 45 polarization by rotating the spin system back into singlet or triplet eigenstates. This step is necessary since the current changes monitored in pEDMR are sensitive to the singlet triplet symmetry of the spin-pair. An elegant way to separate spin-dependent and spin-independent mw induced electrical responses, is provided by ${ }^{50}$ phase cycling of the projection pulse used for spin state readout. $^{101}$

Echo detected pEDMR experiments set the basis for a large variety of pEDMR experiments, like the electrically detected electron spin echo envelope modulation (EDESEEM) (Fig. 3 e) ${ }^{37}$, ${ }_{55}^{50}$ as well as pulsed electrically detected electron nuclear double resonance (EDENDOR) (Fig. $3 \mathrm{f}$ )..$^{33,36}$

EDESEEM is based on the same $\pi / 2-\tau_{1}-\pi-\tau_{2}$ - echo pulse sequence where coherences are refocused at $\tau_{1}=\tau_{2}$ to generate a spin echo, whose amplitude is measured as a function of $\tau=\tau_{2}$.
60 (see Fig. 3 e). Nuclear spins coupled to the electron spin give rise to modulations imprinted on the echo decay. The magnitude of HFI couplings may be determined by Fast Fourier Transformation (FFT) of the echo decay function.

The EDENDOR sequence depicted in Fig. $3 \mathrm{f}$ ) is based on the ${ }_{65}$ Davies ENDOR sequence, ${ }^{102}$ routinely employed in mw detected EPR. The major difference to pEDMR sequences depicted in Fig. 3 c) to e) consists in a radiofrequency (rf) pulse that is applied alongside with the mw pulses. The sequence starts with a mw preparation $\pi$ pulse inverting the population of an electron spin 70 transition in resonance followed by a rf $\pi$ pulse. The effect of the rf pulse on the electron spin echo is monitored by the final $\pi / 2$ $\tau-\pi-\tau-\pi / 2$ readout sequence. If the rf $\pi$ pulse is not in resonance with any nuclear spin coupled to the electron spin the depicted pulse sequence results in a negative echo. In the case 75 where the rf pulse is in resonance with a nuclear spin, the detected echo intensity will be less negative. To obtain the resonance frequency of the coupled nuclei the inverted echo is recorded as a function of the rf. The resulting spectrum is the NMR spectrum of the nuclear spins in the vicinity of the electron. 80 Very recently it was shown that by combining time-programmed light excitation with EDENDOR, selective excitation of ionized $\mathrm{P}$ donor spins in Si with ultra high sensitivity ( 3000 nuclear spins) can be achieved. ${ }^{103}$ EDENDOR and EDESEEM are capable of resolving small hyperfine couplings of electron spins to nearby 85 nuclear spins that are unresolved in the EDMR spectrum. By measuring these couplings the electron spin density distribution of the paramagnetic site under investigation may be mapped out, which provides important structural information about the orbital symmetry and the binding environment. Hyperfine spectra in 90 addition contain information about the isotope composition of the investigated material, which may be employed to locate paramagnetic sites in multi layer devices. ${ }^{50}$

\section{2) pEDMR set-up}

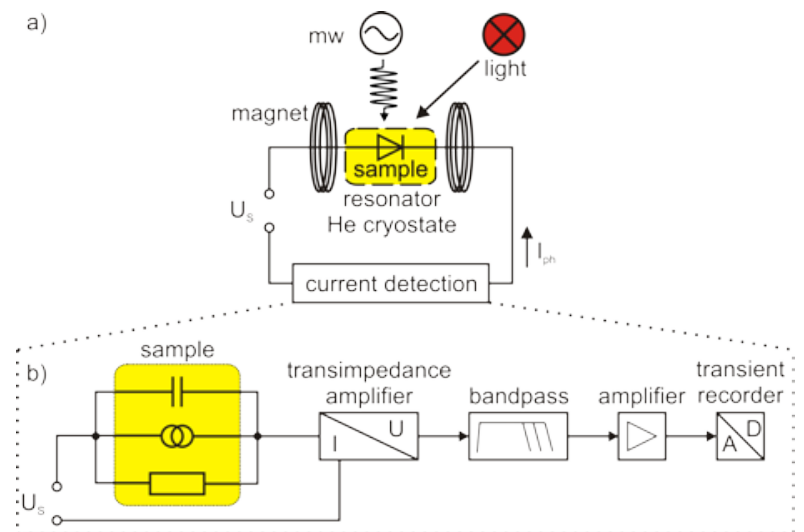

Fig. 4 a) pEDMR setup. The solar cell sample (see e.g. Fig. 5) is located 95 in a He cooled mw resonator . pEDMR excitation is achieved by applying short high-power mw pulses (10-100 ns, $\left.P_{\mathrm{mw}}=1 \mathrm{~kW}\right)$ resonant with an external magnetic field. Sample illumination is achieved via optical windows in the sample cryostat and the resonator or via optical fibres attached to the sample. A constant voltage source is used to apply a 100 voltage to the sample and thus establishing a steady-state (photo-) current $I$. Changes of the current, $\Delta|I|$, are recorded as a function of time by a current detection unit shown in b).

Fig. 4 a) depicts the X-band pEDMR setup at HZB based on a 
commercial EPR spectrometer (Bruker Elexsys E580), which was upgraded by the equipment for electrical detection (see Fig. 4 b)). ${ }^{49}$ The sample is mounted in a dielectric mw resonator (Bruker ER 4118XMD5 or EN 4118XMD4) inside the sample magnet 5 and cooled using a continuous flow helium cryostat (Bruker ER 4118CF) with optical access. Mw excitation is achieved by mw pulses (typical pulse length 10-500 ns) generated by the mw bridge of the EPR spectrometer and amplified by a travelling wave tube amplifier (output power $\sim 1 \mathrm{KW}$ ). The temperature is 10 adjusted between 2.5 and $300 \mathrm{~K}$ by a temperature controller (Oxford ITC 503). Inside the resonator the solar cell samples can be illuminated by a halogen cold light source (Schott KL 2500 LCD), an Argon ion laser (Coherent Innova 90C-A4) or an actively stabilized laser diode $(\lambda=635 \mathrm{~nm}$, Elektronik15 Manufaktur Mahlsdorf) directly through the cryostat window. Alternatively, the light can be fed through a fibre with an attached micro prism that allows for illumination independent of the sample orientation. Fig. 4 b) depicts the current detection scheme. The equivalent circuitry of the solar cell can be considered as a 20 parallel circuit consisting of current source, resistor, and capacitor. A differential transimpedance amplifier (ElektronikManufaktur Mahlsdorf) converts the current signal $\Delta|I|$ into a voltage which is then filtered by a bandpass with lower cut-off frequency of $1 \mathrm{~Hz}$ to remove the DC-component of $I$. The upper 25 cut-off frequency can be varied in order to control the time resolution of the detection setup. ${ }^{49}$ The resulting signal is then amplified to a level that is suitable for the transient recorder (Bruker SpecJet).

\section{3) pEDMR contact scheme}

30 The fabrication of appropriate pEDMR solar cell samples is challenging since the necessary preparation steps differ and have to be optimized for each material system. However, there are general requirements the sample has to fulfil.

Firstly the geometry of solar cells to be analysed is inherently 35 limited by the dimensions of the mw resonator, which, are determined by the wavelength of the mw. At X-band frequencies a solar cell area of $1 \times 1 \mathrm{~mm}^{2}$ has proved to be reasonably small to yield homogeneous mw amplitudes over the whole active area of the solar cell. Secondly, each type of sample requires a special 40 contact structure, which is usually realized in the following way.

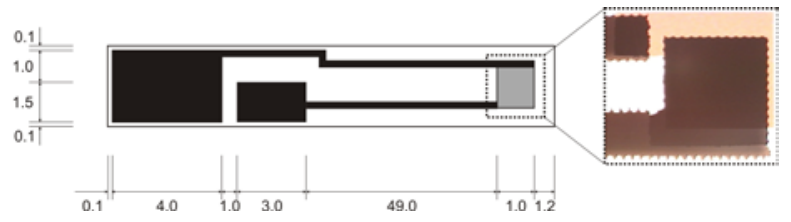

Fig. 5 TFS solar cell contact scheme for pEDMR measurements. The black areas indicate the strip lines and contact pads consisting of 100 to $200 \mathrm{~nm}$ aluminium, gold or silver. The isolating glass substrate is not part of the cell and the contacts run in parallel on the front side of the 45 substrate. To allow for illumination through the transparent substrate $\mathrm{ZnO}$ serves as transparent conductive oxide. The grey rectangles represent the active area (solar cell). Current flows perpendicular to the substrate plane through the multilayer TFS structure. A micrograph from this part of the samples is shown in the blow-up on the right. All dimensions are in $\mathrm{mm}$. 50 Similar contact schemes were employed for pEDMR measurements on OPV cells.

The electrical contacts of the active device (or layer) are connected to contact pads on the opposite side of the EDMR structure via thin film metal stripes on the substrate. Since 55 conductive material generally distorts the mw modes in the resonator and thus leads to inhomogenities of $B_{1}$, it is essential to keep the thickness of the strip lines below the skin depth for the $\mathrm{mw}$ at the particular frequency (approx. $1 \mu \mathrm{m}$ for aluminium at $10 \mathrm{GHz}$ ). For the same reason the strip lines have to be long 60 enough so that the contact pads and the cables connected to them with silver paste are situated outside of the resonator.

Fig. 5 shows the contact structure prepared for pEDMR measurements on $\mu \mathrm{c}-\mathrm{Si}: \mathrm{H}$ solar cells, presented in Section 4. The solar cell (grey area) is electrically connected to contact pads that 65 are positioned outside the mw resonator by metal strip lines (shown in black) with a thickness below the skin depth of the mw radiation. The patterning of the contacts as well as the confinement of the active solar cell area to $1 \mathrm{~mm}^{2}$ was realized by laser scribing techniques. ${ }^{104}$

70 The contact structure is depicted along with a micrograph of the active device areas. This structure is used for thin-film solar cells which are deposited on a foreign substrate (e.g. glass or quartz). Since the (isolating) substrate is not part of the cell, both the front and the rear contact run in parallel on the front side. A similar 75 structure incorporating MEH-PPV/PCBM blends was processed for experiments presented in Section 5.

\section{4. pEDMR on a-Si:H/Hc-Si:H solar cells}

Solar cells based on a-Si:H and $\mu \mathrm{c}-\mathrm{Si}: \mathrm{H}$ offer the potential for a significant energy and cost reduction, as they can be deposited at 80 moderate temperatures on inexpensive substrates ${ }^{105}$ with low material consumption. High-quality $\mu \mathrm{c}-\mathrm{Si}: \mathrm{H}$ consisting of small crystallites surrounded by an amorphous phase and device grade a-Si:H can be combined in tandem solar cells by plasma enhanced chemical vapour deposition (PECVD) processes. ${ }^{106}$ 85 Due to their complementary electronic and optical properties efficient use of the solar spectrum ${ }^{107}$ can be accompanied by a significant reduction of light-induced degradation of the electronic quality. ${ }^{108}$

Still, TFS solar cells suffer from an inferior electronic quality 90 compared to crystalline silicon. In thin-film devices trapping and recombination at localized defects in the band gap and tail states near the band edges are the main limiting factors for the cell performance. $^{21,81,109-111}$ Loss of device performance in TFS can occur in the bulk of the absorber layers due to material disorder 95 which is induced by the fabrication process, ${ }^{21,112}$ at the homo and heterojunction interfaces to the doped and window layers ${ }^{113}$ as well as a result of material degradation. ${ }^{114}$ Many of the deviceperformance determining defects are paramagnetic (in the case of $\mathrm{db}$ ) or can be made paramagnetic by light excitation (in the case 100 of band-tails), which renders EPR ideally suited to study defect concentrations and the local defect environment. ${ }^{110,112,115,116}$ In recent years we developed and applied dedicated pEDMR methods (see Fig. 3) to study the impact of structural defects in fully processed TFS solar cells. ${ }^{26,} 28,48-50,53$ In order to 105 demonstrate the potential of pEDMR to identify and track device limiting spin-dependent transport and loss mechanisms in TFS solar cells we will in the following discuss some of our recent achievements on $\mu \mathrm{c}-\mathrm{Si}: \mathrm{H}$ thin-film solar cells. To improve charge carrier collection these devices are fabricated in the pin 110 configuration. Details of the device architecture are depicted in Fig. 6. Note that the highly p-doped $\mu \mathrm{c}-\mathrm{Si}: \mathrm{H}$ is used as a window layer which has superior quality as compared to a p-doped a-Si:H 
layer.

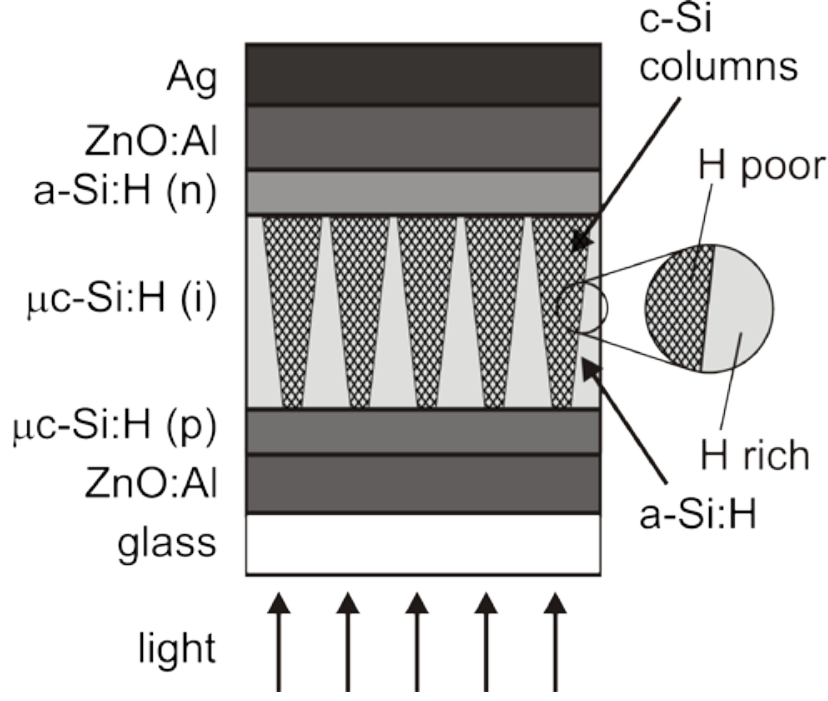

Fig. 6 Left: Layer structure of the superstrate thin-film n-a-Si:H/i- $\mu \mathrm{c}-$ $\mathrm{Si}: \mathrm{H} / \mathrm{p}-\mu \mathrm{c}-\mathrm{Si}: \mathrm{H}$ pin solar cell. Right: Enlarged view of the phase boundary between $\mathrm{H}$ rich a-Si:H and $\mathrm{H}$ depleted c-Si grains in the $\mu \mathrm{c}-\mathrm{Si}$ material.

$5 \mu \mathrm{c}-\mathrm{Si}: \mathrm{H}$ solar cells were deposited at the Forschungszentrum Jülich by PECVD on Corning glass ${ }^{106}$ with a process that was shown to reach conversion efficiencies above $10 \%{ }^{117}$ Fig. 6 depicts the layer structure of the solar cells, consisting of $150 \mathrm{~nm}$ $\mathrm{ZnO}: \mathrm{Al}$ TCO, boron-doped $\mathrm{p}-\mu \mathrm{c}-\mathrm{Si}: \mathrm{H}, 1 \mu \mathrm{m}$ intrinsic $\mu \mathrm{c}-\mathrm{Si}: \mathrm{H}$ 10 absorber, phosphorous-doped n-a-Si:H emitter, $\mathrm{ZnO}$ :Al and silver as a back contact. The total phosphorus and boron concentration in the films is estimated to be 1.5 at. \% and 0.2 at. \%, respectively. The solid-phase hydrogen concentration in $\mu \mathrm{c}-\mathrm{Si}: \mathrm{H}$ and a-Si:H is known to be about 10 at. \%. pEDMR measurements 15 were carried out on the pEDMR set-up described in Section 3.2. The solar cell was operated in reverse direction $\left(U_{\text {bias }}=-1.0 \mathrm{~V}\right)$ and cooled to a temperature of $10 \mathrm{~K}$ by a He-flow cryostat resulting in a photocurrent of $17 \mu \mathrm{A}$ (active layer area of about 1 $\mathrm{mm}^{2}$ ) under illumination with a heat-filtered tungsten halogen 20 lamp (50 mW/ $\left.\mathrm{cm}^{2}\right)$.

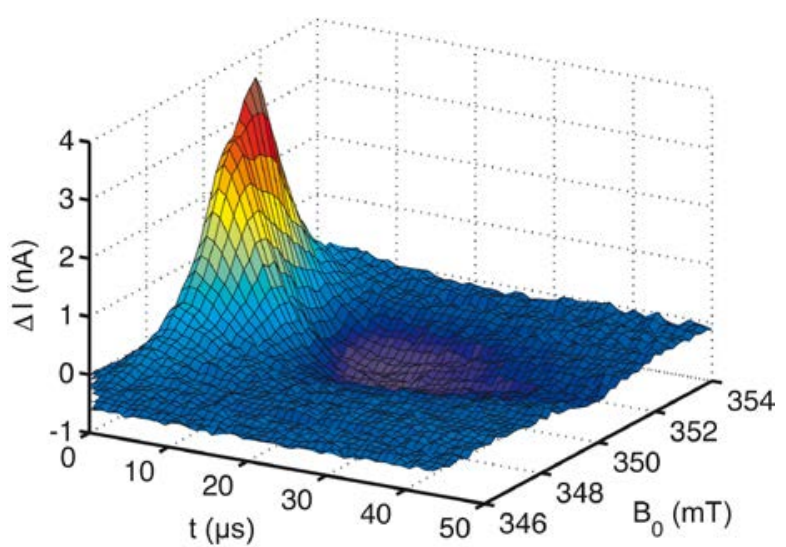

Fig. 7 2D pEDMR spectrum of a $\mu \mathrm{c}-\mathrm{Si}: \mathrm{H}$ pin solar cell measured at $T=10 \mathrm{~K}$. The sample was biased in reverse direction and illuminated with white light. $\Delta I$ was detected after a $320 \mathrm{~ns}$ long mw pulse $\left(f_{\mathrm{mw}}=\right.$ $259.81 \mathrm{GHz})$.

Fig. 7 depicts a 2 dimensional (2D) pEDMR spectrum of a $\mu \mathrm{c}-$ Si:H pin solar cell at $T=10 \mathrm{~K}$ showing transient current changes $\Delta I$ as a function of time after the mw excitation pulse $\left(\tau_{\mathrm{P}}=320\right.$ ns) and the external magnetic field $B_{0}$ (see also Fig. 3a)). The 30 spectrum exhibits an asymmetric resonance line with a pronounced field dependence of the current transients. Both facts indicate the presence of different EDMR signals contributing to the observed pEDMR spectrum.

In the following we will demonstrate how advanced pEDMR 35 detection schemes may be employed to deconvolute the different spectral components and conclude on their nanoscopic environment as well as the underlying spin-dependent transport processes and locate them in the multilayer solar-cell (see Fig. 6).

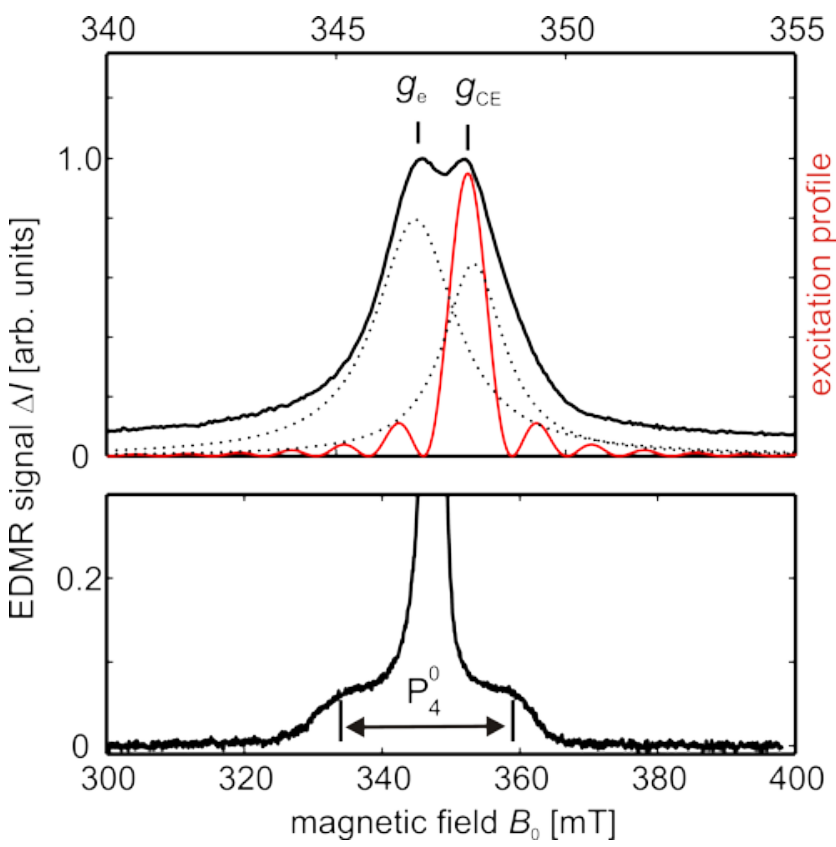

Fig. 8 Top: pEDMR spectrum ( $\Delta I$ normalized to 1 ) of a $\mu c-S i: H$ pin solar 40 cell taken $2.2 \mu$ s after a $100 \mathrm{~ns} \mathrm{mw}$ pulse, $T=10 \mathrm{~K}$ (fat solid line: experiment; dotted lines: individual spectral components as obtained from the fit, thin red solid line: excitation profile of the mw pulse). Resonance positions of the different paramagnetic sites are indicated by vertical lines. Bottom: pEDMR spectrum on a broader magnetic field range 45 measured under similar experimental conditions optimized for this field range.

Fig. 8 shows field slices of the 2D pEDMR spectrum recorded $2.2 \mu$ s after a $100 \mathrm{~ns}$ mw $\pi$-pulse (top) and the same spectrum on a broader field range (bottom) measured under the same 50 experimental conditions except for a mw power increased by $9 \mathrm{~dB}$ in order to enhance the broad spectral components. The spectra contain two narrow components and, in addition, a broad (> $20 \mathrm{mT}$ ) component with lower intensity. For the identification of the paramagnetic sites we analysed the pEDMR spectrum by 55 fitting Lorentzian and Gaussian functions to the experimental data. Best fits to the experimental spectra can be obtained when assuming two narrow resonances at $g_{\mathrm{CE}}=1.9975(5)$ and $g_{\mathrm{e}}=2.0049$ (5) and an additional pair of lines separated by $25 \mathrm{mT}$, which are symmetric to $g_{\mathrm{P}}=2.003$. This pronounced splitting is 60 well known to arise from hyperfine interaction between phosphorus electron and nuclear spins in a-Si:H. ${ }^{118}$ In the present sample structure (see Fig. 6) phosphorus is only incorporated in the n-doped a-Si:H window layer. Moreover, for $\mu \mathrm{c}-\mathrm{Si}: \mathrm{H}$ neither the $4.2 \mathrm{mT}$ hyperfine splitting known from c-Si, nor the 65 abovementioned $25 \mathrm{mT}$ are observed even when the material is 
deliberately doped with $\mathrm{P} .{ }^{21,} 110$ These facts allow for an assignment of the broad signal component to paramagnetic fourfold-coordinated $\mathrm{P}_{4}^{0}$ donor atoms in the n-a-Si:H layer. ${ }^{49}$ In intrinsic and n-doped $\mu \mathrm{c}-\mathrm{Si}: \mathrm{H}$ an EPR resonance at $g=1.997-$ 51.998 was observed and assigned to shallow localised states in energetic proximity to $E_{\mathrm{C}}$, typically referred to as CE states. ${ }^{110}$, 119-121 It was demonstrated by cwEDMR measurements on $\mu \mathrm{c}$ $\mathrm{Si}: \mathrm{H}$ films that these states are involved in hopping-transport at low temperatures. ${ }^{23,122}$ The $g$ value of the second resonance, $g_{\mathrm{e}}$, is 10 close to $g=2.0044$, which is the signature of an EDMR signal which was recently associated with hopping of electrons between band-tail states in a-Si:H (known as the 'e signal'). ${ }^{123,}{ }^{124}$ Therefore $g_{\mathrm{e}}$ is associated with conduction band-tail states in the n-a-Si:H layer. However, additional sources of evidence had to be 15 employed to unambiguously assign the observed EDMR resonances and the underlying transport processes.

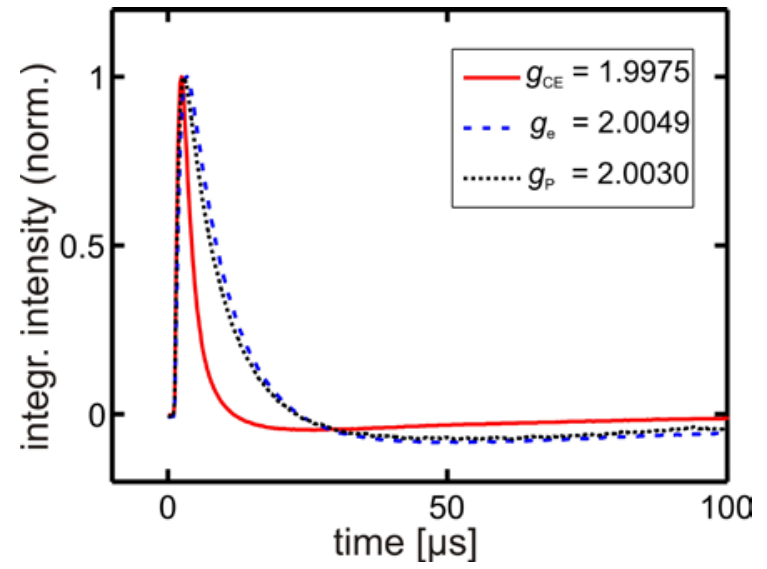

Fig. 9 Integrated peak intensities $\left(A_{\text {peak }}\right)$ obtained by deconvoluting the 2D pEDMR spectrum (Fig. 7) with wide magnetic field range. The broad spectral component was taken into account by assuming a pair of 20 Gaussian lines that are symmetric to $g_{\mathrm{P}}=2.003$. Ordinate scales were normalized to 1 .

EDMR signals are due to altered spin-dependent charge-transfer probabilities between paramagnetic states. In the framework of the KSM model, application of an intense mw pulse, in resonance 25 with either of the participating states, generally increases the transfer probability. However, this rate change of a microscopic process can influence the photocurrent in a solar cell in different ways. It depends in a complicated way on the microscopic mechanism, the device physics, and, in the particular case of pin 30 solar cells, on the bias voltage applied to the sample. ${ }^{115,116}$

Both, the time evolution as well as the sign of $\Delta I$ may be employed to conclude on the character of the observed spindependent charge transport process. To extract this information from the 2D pEDMR data (Fig. 7) we deconvoluted the signals in 35 time domain by taking the line parameters from the spectra shown in Fig. 8 as fixed parameters and their relative intensities as fit parameters. In this way we obtained the integrated peak intensities $A_{\text {peak }}$ of the resonances at $g_{\mathrm{CE}}=1.9975(5)$ $(\mathrm{FWHM}=1.8 \mathrm{mT})$ and $g_{\mathrm{e}}=2.0049(5)(\mathrm{FWHM}=1.8 \mathrm{mT})$. The 40 signal at $g_{\mathrm{P}}=2.003$ was taken account of by assuming a pair of symmetric Gaussian lines centred around $g_{\mathrm{P}}$ with equal intensities split by $25 \mathrm{mT}$. Thus, we were able to independently study the time behaviour of the spectrally overlapping signals. Fig. 9 shows the results of this analysis. The intensity axes are normalised to 45 allow for a good comparison between the dynamics of the transient signals.
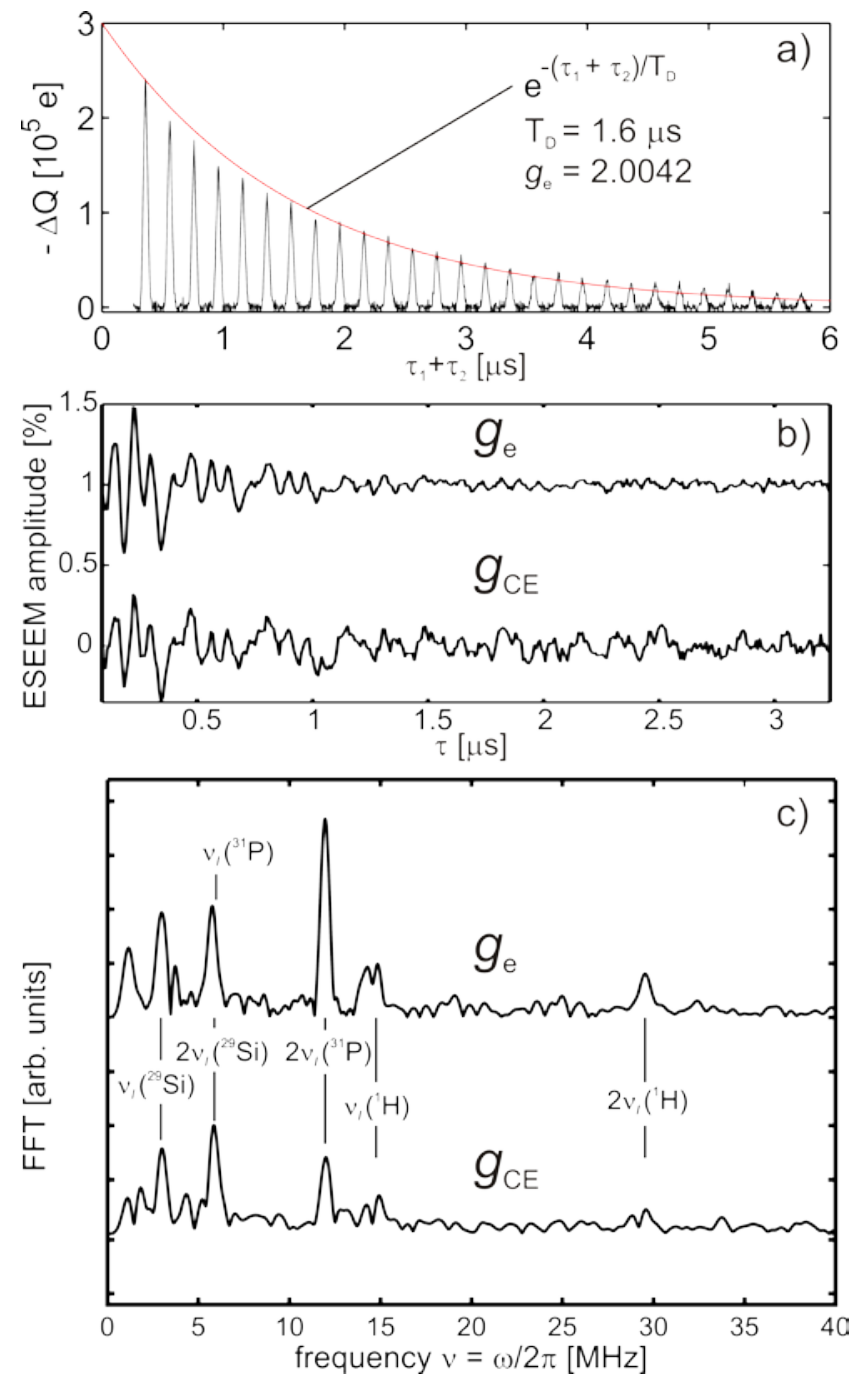

Fig. 10 a) Electrically detected electron spin echoes measured on the $g_{\mathrm{e}}$ resonance position. Spin echoes were recorded with the pulse sequence given in Fig. 3 e) by simultaneously incrementing $\tau_{1}$ and $\tau_{2}$. The echo 50 decays approximately mono-exponentially (time constant $T_{\mathrm{D}}=1.6 \mu \mathrm{s}$ ). ESEEM modulations are superimposed on the echo decay. b) EDESEEM measured. at $g_{\mathrm{e}}$ (upper trace) and $g_{\mathrm{CE}}$ (lower trace). The displayed time traces were obtained by dividing the raw data by a $9^{\text {th }}$-order polynomial fit. a) Magnitude FFT spectra of echo modulations in a) normalized to ${ }_{55} 2 \omega_{\mathrm{L}}\left({ }^{29} \mathrm{Si}\right)$ modulation. Vertical lines indicate the nuclear Larmor frequencies $\left(\omega_{\mathrm{L}}\right)$ of ${ }^{29} \mathrm{Si},{ }^{31} \mathrm{P}$, and ${ }^{1} \mathrm{H}$, respectively

The present pEDMR data has been recorded in reverse bias. Under this condition we found for all three signals an enhancement of the photocurrent $(\Delta I>0)$ immediately after the $60 \mathrm{mw}$ pulse. The positive sign of $\Delta I$ is an indication for a spindependent hopping process, whereas one would expect an initial quenching $(\Delta I<0)$ right after the mw pulse in the case of spindependent recombination. Note that in both cases theory predicts that the pEDMR transient shows a sign reversal when non65 vanishing triplet transition probabilities are present. $^{28,} 80$ Additional information, whether the pEDMR signals originate from one spin-dependent transport process or whether different mechanisms give rise to the $\mathrm{PEDMR}$ resonances may be obtained from the time dependence of the pEDMR signals. In the present 70 case the time dependence of the pEDMR transients at $g_{\mathrm{CE}}$ and $\mathrm{g}_{\mathrm{e}}$ 
strongly differs, whereas a clear correlation between the rise and fall times of $g_{\mathrm{e}}$ and $g_{\mathrm{p}}$ may be observed. For this to occur, both centres must either be involved in one process (namely hopping from phosphorus to a tail state and vice versa) or two different 5 processes (hopping via phosphorus states and hopping via bandtail states) which influence $I_{\mathrm{ph}}$ in a similar way. ${ }^{41}$ The latter process seems to be very unlikely, considering the large difference in density of states. Based on the experimental results we cannot unambiguously discriminate between both ${ }_{10}$ possibilities, but the correlation between the pEDMR transients supports the interpretation that both signals stem from a spindependent hopping process in the $\mathrm{n}-\mathrm{a}-\mathrm{Si}: \mathrm{H}$ layer. To further corroborate this assignment and conclude on the origin of the $\mathrm{CE}$ signal we performed EDESEEM measurements on the same 15 sample structure.

Since the online monitoring of electron-spin coherence as in conventional EPR is not possible in EDMR, the electron-spin echo decay depicted in Fig. 10 a) was recorded in a twodimensional experiment with the pulse sequence depicted in Fig. 203 e). ${ }^{37,}{ }^{50}$ Fig 10 b) displays EDESEEM after eliminating the unmodulated part of the echo envelope recorded at resonance positions of the $g_{\mathrm{CE}}$ and $g_{\mathrm{e}}$ EDMR signals (see Fig. 8). The FFT spectra of the respective time traces are depicted in Fig. $10 \mathrm{c}$ ). Several pronounced resonance peaks are observed at frequencies 25 matching the nuclear Larmor frequencies $\omega_{\mathrm{L}}$ or $2 \omega_{\mathrm{L}}$ of ${ }^{29} \mathrm{Si},{ }^{31} \mathrm{P}$ and ${ }^{1} \mathrm{H}$ nuclei, respectively. This indicates that ESEEM originate from abundant distant nuclei with weak anisotropic and negligible isotropic HFI, since only in this case ESEEM frequencies match the nuclear Larmor frequencies.

30 The spectrum recorded at $g_{\mathrm{e}}$ shows strong ${ }^{31} \mathrm{P}$ ESEEM, which further supports the assignment of these centres to the n-a-Si:H emitter layer doped with a large phosphorous concentration. In contrast to crystalline silicon, doping studies of a-Si:H showed that phosphorous atoms occur in threefold- $\left(\mathrm{P}_{3}\right)$ and fourfold35 coordinated $\left(\mathrm{P}_{4}\right)$ atomic configurations, where only the latter acts as a dopant. ${ }^{125}$ Note that only the neutral state $\left(\mathrm{P}_{4}\right)$ is paramagnetic. The ${ }^{31} \mathrm{P}$ nuclei observed by ESEEM can only be attributed to the diamagnetic $\mathrm{P}_{3}{ }^{0}$ configuration. In the alternative case large HFI between the electron spin of the $\mathrm{P}_{4}{ }^{0}$ configuration ${ }_{40}$ and the ${ }^{31} \mathrm{P}$ nuclear spin on the same atom would shift the ESEEM resonances out of the detection window. ${ }^{126}$ This assignment is also supported by the fact that the present spin pairs observed by EDMR are weakly coupled i.e., they exhibit a negligible electron-electron coupling. This indicates that the 45 electron spins are separated by more than $3 \mathrm{~nm}$, a value well beyond the detection radius of ESEEM $(\sim 8 \AA)$.

EDESEEM spectra obtained at $g_{\mathrm{CE}}=1.9983$ show the same resonance positions as at $g_{\mathrm{e}}$ but a strong reduction of the $\omega\left({ }^{31} \mathrm{P}\right) / \omega\left({ }^{29} \mathrm{Si}\right)$ intensity ratio (cf. lower curve Fig. $\left.10 \mathrm{c}\right)$. Reduced

${ }^{50}{ }^{31} \mathrm{P}$ intensity supports the assignment of the $g_{\mathrm{CE}}$ resonance to the $\mu \mathrm{c}-\mathrm{Si}: \mathrm{H}$ layer, which, in contrary to the n-a-Si:H layer, does not contain $\mathrm{P}$ dopants. The residual contribution of ${ }^{31} \mathrm{P}$ ESEEM does not arise from HFI between $\mathrm{CE}$ electron spins and ${ }^{31} \mathrm{P}$ nuclear spins. Instead it may be attributed to an off-resonant excitation of 55 e-centres by the excitation profile of the rectangular mw pulse. This is verified by a numerical simulation of the mw pulse excitation profile (cf. Fig. 8), which indicates that a $32 \mathrm{~ns} \mathrm{mw}$ pulse excites a large fraction of the e-centre resonance. Quantitative simulations reveal that the off-resonant e-centre
60 contribution is only reduced by a factor of two when tuning the magnetic field to $g_{\mathrm{CE}}$, which is in accordance with the observed relative amplitudes of the different isotopes in the ED-ESEEM spectrum (cf. Fig. 10 c).

The same argumentation holds for ${ }^{1} \mathrm{H}$ ESEEM observed at the ${ }_{65}$ resonance position of the $\mathrm{CE}$ centres. The signal contributions at $\omega_{\mathrm{L}}\left({ }^{1} \mathrm{H}\right)$ or $2 \omega_{\mathrm{L}}\left({ }^{1} \mathrm{H}\right)$ can again be assigned to an off-resonant excitation of e-centres as they are reduced by about a factor of two as compared to e-centres. Simulations show that CE centres alone do not show signal contributions at $\omega_{\mathrm{L}}\left({ }^{1} \mathrm{H}\right)$ or $2 \omega_{\mathrm{L}}\left({ }^{1} \mathrm{H}\right),{ }^{50}$

70 hence they are not coupled to distant $\mathrm{H}$ atoms. The fact that the vicinity ( $r<6 \AA$ ) of CE centres is depleted from hydrogen has important consequences for the microscopic assignment of $\mathrm{CE}$ centres in the $\mu \mathrm{c}-\mathrm{Si}: \mathrm{H}$ material. In $\mu \mathrm{c}-\mathrm{Si}: \mathrm{H}$ grains of perfect crystal quality form columns that are separated by grain 75 boundaries (see Fig. 6). ${ }^{127}$ In contrast to a-Si:H, these crystalline grains contain only small amounts of hydrogen. Due to this fact the absence of ${ }^{1} \mathrm{H}$-ESEEM in case of CE centres indicates that CE centres are located inside crystalline columns or grains and not in the hydrogen rich amorphous phase or at the boundary between 80 crystalline and amorphous tissue phases at the surface of the columns (see Fig. 6). However, in $\mu \mathrm{c}-\mathrm{Si}: \mathrm{H}$ dislocations or point defects are absent in crystalline columns and grains. ${ }^{127}$ The dominant lattice distortions or crystal defects in crystalline columns are coherent twin boundaries. ${ }^{127}$ We therefore assign CE 85 centres to localized conduction band-tail states induced by twin grain boundaries. These centres are restricted to the crystalline phase of $\mu \mathrm{c}-\mathrm{Si}: \mathrm{H}$.

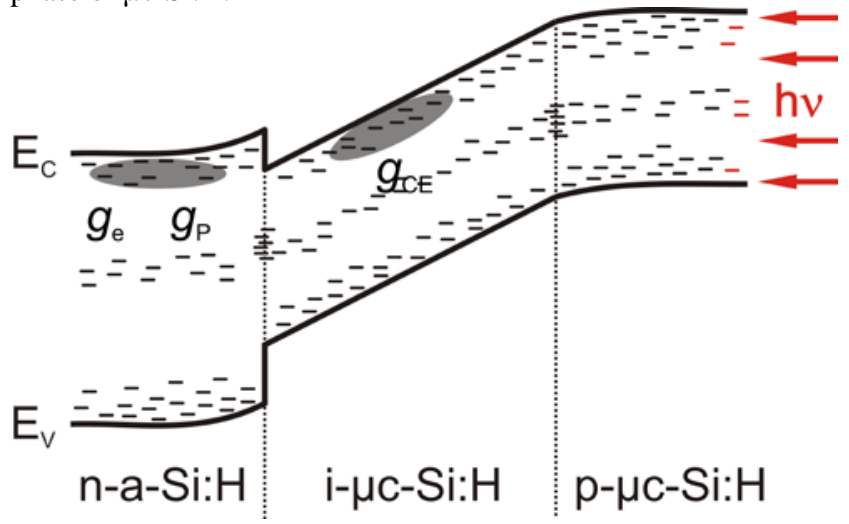

Fig. 11 Band-diagram of a $\mu \mathrm{c}-\mathrm{Si}: \mathrm{H}$ pin solar cell at room temperature. 90 The paramagnetic states giving rise to the pEDMR signals are labelled by their respective g values.

In summary, we demonstrated that the ultra sensitive detection and identification of paramagnetic states as well as their assignment to particular solar cell layers and charge transport 95 processes can be accomplished by pEDMR in completely processed pin solar cells. To separate EDMR signals originating from the respective layers we deconvoluted spectrally overlapping signals in the time domain and complemented this information with EDESEEM experiments selective to different 100 paramagnetic sites in the sample. The paramagnetic centres that were identified by pEDMR are sketched in Fig. 11 and labelled by their respective g-values. These states lead to the following spin-dependent transport processes. In the n-a-Si:H window layer electron hopping between conduction band-tail states and 105 phosphorus donor states dominates at cryogenic temperatures. 
Similarly, in the intrinsic $\mu \mathrm{c}-\mathrm{Si}: \mathrm{H}$ absorber hopping occurs via paramagnetic conduction band-tail states that could be traced back to twin boundaries inside crystalline grains. The above mentioned measurements were performed at cryogenic 5 temperatures. The identification of defect states and transport mechanisms at low temperatures constitutes the basis for studies focusing on spin-dependent processes under normal solar cell operating conditions. Benefiting from instrumental improvements we succeeded in performing pEDMR measurements 10 (photocurrent relaxation as well as coherent experiments) at room temperature.

Fig 12 (a) shows first pEDMR measurements on $\mu c-S i: H$ pin solar cells performed at $T=300 \mathrm{~K}$ in the dark under forward bias injection at $U=0.47 \mathrm{~V}$. We observe a positive asymmetric 15 pEDMR signal at $g=2.0059$ close to the signature that is generally associated with dbs in intrinsic $\mu \mathrm{c}-\mathrm{Si}: \mathrm{H}$. The inset of Fig. 12 shows the spectrum on a broader magnetic field range. It was measured under the same experimental conditions except for a mw power increased by $9 \mathrm{~dB}$ to accentuate possible broad 20 spectral components. In contrast to the low temperature case (see Fig. 8) we observe no contributions from phosphorus states in the $\mathrm{n}$-a-Si:H or any other broad features. While at $10 \mathrm{~K}$ hoppingtransport among tail states prevails, the conductivity at $300 \mathrm{~K}$ is dominated by transport that takes place above the mobility edge 25 and therefore does not exhibit the typical spin-dependence of hopping-transport. These arguments support the assumption that the pEDMR signal originates from recombination of electrically injected electrons and holes via db states in the i- $\mu \mathrm{c}-\mathrm{Si}: \mathrm{H}$ layer. This is in agreement with previous cwEDMR measurements and 30 simulations on $\mu \mathrm{c}-\mathrm{Si}: \mathrm{H}$ pin solar cells. ${ }^{12,}{ }^{128}$ However, based on the pEDMR spectra alone it is impossible to elucidate the underlying microscopic mechanism of this spin-dependent process. In order to shed additional light on the underlying spindependent transport process, we performed room temperature 35 Rabi oscillation measurements at the $g=2.0059$ resonance peak [cf. Fig. 12(b)] of $\mu \mathrm{c}-\mathrm{Si}: \mathrm{H}$ pin solar cells. Oscillations could be recorded by varying the mw pulse length for different mw power levels. We observed a single Rabi nutation frequency that increases with increasing mw power. The fact that the double ${ }_{40}$ Rabi frequency is missing indicates that the room temperature spin nutations of $\mu \mathrm{c}-\mathrm{Si}: \mathrm{H}$ pin solar cells are dominated by the Rabi frequency of spin pairs under selective excitation conditions (see next Section for a detailed description). Under the assumption of a KSM process, this means that only one spin-pair ${ }_{45}$ partner is excited by the mw pulse. The reason for this result is not clear yet, but it clearly shows that the observed room temperature EDMR signal is not associated with a direct capture process. Hence, further experiments are necessary to identify the spin-dependent process observed at room temperature.

${ }_{50}$ We are convinced that advanced pEDMR techniques will impact the further development of Si solar cells. Just to mention two important examples. Firstly, the further development of thin-film solar cells based on polycrystalline (poly)-Si with grains larger 1 $\mu \mathrm{m}$ with higher charge carrier mobility and smaller grain 55 boundary surfaces area. ${ }^{129-131}$ Such materials can be obtained by exposing evaporated a-Si samples on glass in an oven to a temperature of $600{ }^{\circ} \mathrm{C}$ for about 24 hours (solid phase crystallization, SPC) or by melting the a-Si layer with a laser or electron beam. Although such techniques may produce grain 60 sizes larger than $20 \mu \mathrm{m}$, the resulting $V_{\mathrm{OC}}$ of poly-Si solar cells is smaller than that of $\mu \mathrm{c}-\mathrm{Si}: \mathrm{H}^{132,133}$ The reason for this observation is currently believed to originate from the poor passivation of defects at the poly-Si grain boundary, which can only be realized by a post deposition hydrogenation treatment. ${ }^{134}$
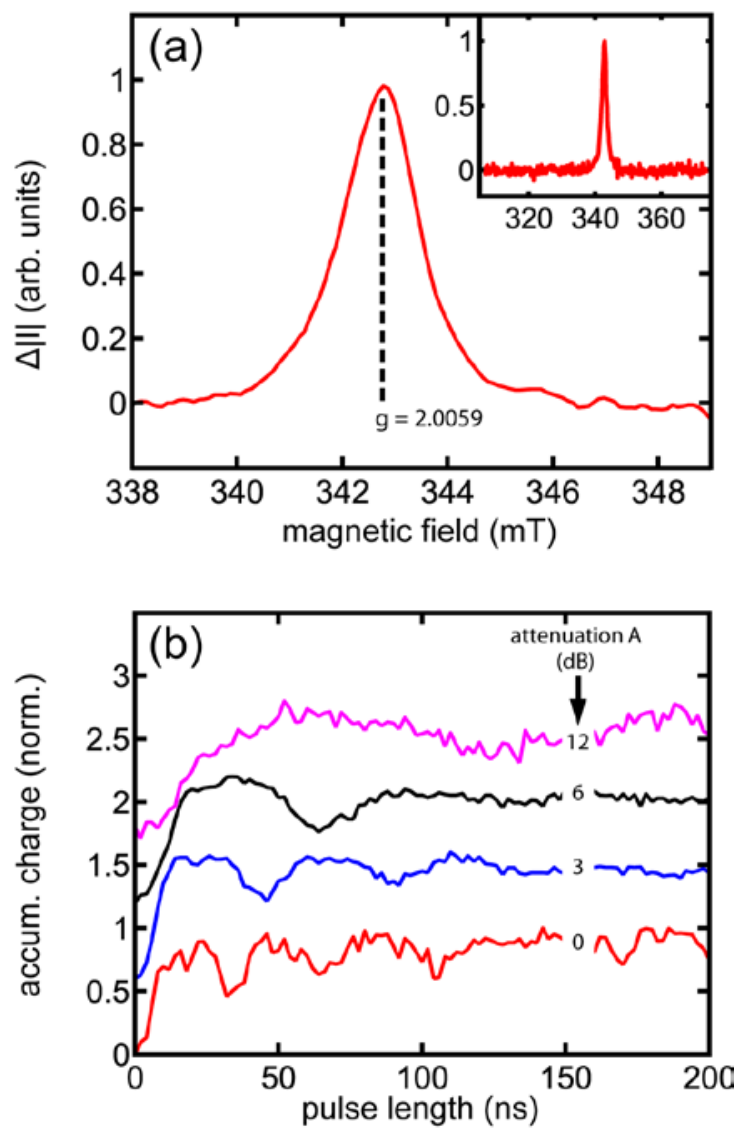

Fig. 12 (a) Room-temperature pEDMR spectrum taken at $t=2.0 \mu \mathrm{s}$ after a $320 \mathrm{~ns}$ long $\mathrm{mw}$ pulse. The measurement was performed without illumination in the forward bias regime $(U=0.47 \mathrm{~V}, I=20 \mu \mathrm{A})$. The inset shows the pEDMR spectrum on a larger magnetic field range. (b) 70 Electrically detected spin-Rabi nutations at the centre of the resonance line at $g=2.0059$ for several mw attenuation levels, $A$, as indicated in the plot.

Secondly, the further improvement of wafer-based c-Si solar cells by reducing charge carrier recombination at the surface or 75 backside of the c-Si absorber, for instance by depositing ultra-thin layers of doped a-Si:H $(10 \mathrm{~nm})$ at temperatures as low as $150^{\circ}$ $200^{\circ} \mathrm{C}$. If properly doped, this a-Si:H layer forms a pn heterojunction at the front and a nn+ heterojunction at the backside of the solar cell which act as minority and majority 80 charge carrier blocking and surface passivation layer, respectively. The growth of these layers only involves low temperatures and module efficiencies as high as $23 \%$ have been reported on (100)-oriented wafers, if an intrinsic a-Si:H buffer layer is deposited prior to the junction-forming doped a-Si:H ${ }_{85}$ layer. ${ }^{135}$ However, in order to further increase the cell efficiency beyond $23 \%$, a detailed understanding of spin dependent and spin independent charge carrier trapping through interface states is required.

In both cases pEDMR is capable of answering remaining 90 questions about the ongoing charge transport and loss mechanisms and thereby assist the design of novel solar cells with optimum efficiency.

The potential of pEDMR techniques is not limited to TFS, c-Si 
and its alloys, but may be useful also for the study of other PV materials like CIS, CIGS or CdTe. First attempts to detect an EDMR signal in CIGS solar cells by our group have so far not been successful. Nevertheless, new efforts in this unexplored area 5 of research may provide useful information about transport and loss mechanisms in solar cells incorporating different semiconductor materials.

\section{5. pEDMR on MEH-PPV/PCBM solar cells}

Optoelectronic devices based on organic semiconductors (OSC) 10 are subject to intense research activities. They provide the possibility to combine low cost and ease of production and are thus qualified to set the stage for cheap electronic applications. In the display market optoelectronic components based on OSC were shown to outperform their inorganic counterparts in many 15 aspects and by now found their way into several commercial applications. A similar development can be expected in the field of OPV, where bringing down the costs for solar energy conversion is the main obstacle on the way towards making solar energy conversion compatible with conventional energy sources. 20 In fact, the maximum efficiency of organic solar cells has steeply increased in recent years. ${ }^{132}$

Common to all devices made from OSC is that they critically depend on a detailed knowledge of charge transport in the composing materials. In Organic Light Emitting Diodes (OLED) 25 the charges must efficiently be transported to the place where they recombine radiatively, in OPV cells the separated positive and negative charge carriers forming upon exciton dissociation (cf. Sec. 2) have to be transported towards the electrodes without losses. In contrast to many inorganic semiconductors, the spin 30 degree of freedom in OSC plays an important role even at room temperature. The spin-orbit coupling in organic materials is low, resulting in long spin-relaxation and spin-coherence times. ${ }^{136}$ Further, conduction in OSC is usually dominated by hopping of charge carriers between localized states. Bound excited states 35 (excitons) may influence charge transport more severely than in classical semiconductors because of the relatively low dielectric constant, which translates into strong exciton binding energies. Despite the important consequences of the spin on charge transport in OSC, details of the relevant transport processes, in 40 particular those influenced by spin selection rules, remain elusive to date. EDMR is well suited to provide a detailed understanding of the interrelation between localized charge carriers and the conductivity in OPV devices. As explained in Sec. 2, this technique is particularly sensitive to spin-dependent 45 recombination and hopping-transport involving doubly occupied sites (bipolarons). Both processes have previously been investigated using $\mathrm{cwEDMR}^{72,137-140}$ and originate from the same spin-dependent processes as discussed before in case of a-Si:H and $\mu \mathrm{c}-\mathrm{Si}: \mathrm{H}$. However, in many cases the cwEDMR spectrum 50 alone does not provide sufficient insight into the detailed microscopic mechanisms because different spin-dependent processes may result in indistinguishable spectra. For example, when the EDMR spectrum exhibits a structureless line consistent with the EPR signature of e.g. $\mathrm{P}^{+}$, several possible scenarios must 55 be considered. In this case the EDMR signal may equally arise from (i) recombination of positive and negative polarons when the resonance line associated with $\mathrm{P}^{-}$is either too broad to be visible in the spectrum or indistinguishable from the spectrum of the positive polaron, (ii) spin-dependent formation of bipolarons 60 from weakly coupled $\mathrm{P}^{+} \mathrm{P}^{+}$precursor pairs, (iii) quenching of triplet excitons by positive polarons or (iv) spin-dependent processes involving a single $\mathrm{P}^{+}$only. In many cases some of the above scenarios can be excluded based on a careful evaluation of the experimental conditions and knowledge about charge 65 transport obtained from electrical characterisation of the sample. Nevertheless, determining the microscopic process governing the EDMR spectrum is often plagued with ambiguity.

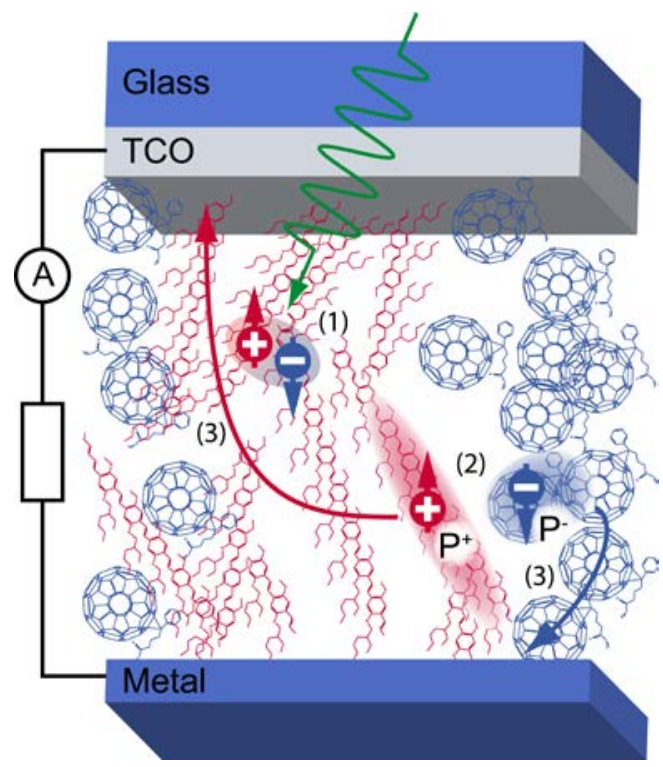

Fig. 13 Bulk heterojunction (BHJ) solar cell structure for EDMR 70 measurements. MEH-PPV and PCBM molecules are shown in red and blue, respectively. (1) During solar cell operation, light excited $\mathrm{P}^{+}$(red spheres) and $\mathrm{P}^{-}$(blue spheres) with random spin orientation (indicated by short arrows) are created and (2) separated at the interface between electron and hole conducting regions. $\mathrm{P}^{+}$and $\mathrm{P}^{-}$are than extracted via 75 MEH-PPV and PCBM phases, respectively. Polaron pathways are indicated by long arrows. Instead of measuring the effect of light excitation, the fate of polarons in OPV materials may be studied by selective injection of charges by a bias voltage bias (polaron pathways not shown).

80 pEDMR can help because apart from delivering the spectral fingerprint of the contributing paramagnetic centres this technique can provide additional information on the spindependent mechanism in a wide range of materials and material combinations.

${ }_{85}$ Recently, pEDMR was used to investigate recombination of photogenerated $\mathrm{P}^{+} \mathrm{P}^{-}$pairs in films of poly(2-methoxy-5-(2'ethyl)-hexyloxy-p-phenylene) vinylene (MEH-PPV) at low temperature. $^{95}$ The hyperfine-field experienced by the recombining $\mathrm{P}^{+} \mathrm{P}^{-}$pairs was explored by exploiting coherent spin 90 effects. ${ }^{141}$ Yet, the application of pEDMR is not restricted to the low-temperature regime, making this technique particularly useful for the investigation of charge transport in solar cells under realistic solar cell operating conditions. We have applied pEDMR to study charge transport through the conjugated polymer $\mathrm{MEH}$ ${ }_{95} \mathrm{PPV}$ in BHJ solar cells using [6,6]-phenyl $\mathrm{C}_{61}$-butyric acid methyl ester (PCBM) as acceptor material. The solar cells were fabricated on indium tin oxide (ITO) coated glass substrates. After cleaning and structuring the ITO electrodes, a single layer of MEH-PPV and PCBM was spin-coated from a solution in 
chlorobenzene in the ratio of 1:4 (by weight). A $100 \mathrm{~nm}$ thick $\mathrm{Al}$ layer was subsequently deposited through a shadow mask, giving an active device area of $5 \mathrm{~mm}^{2}$. The electrodes extended along the length of the substrate to bring them out of the mw resonator. 5 The structure of the solar cells is depicted in Fig. 13.
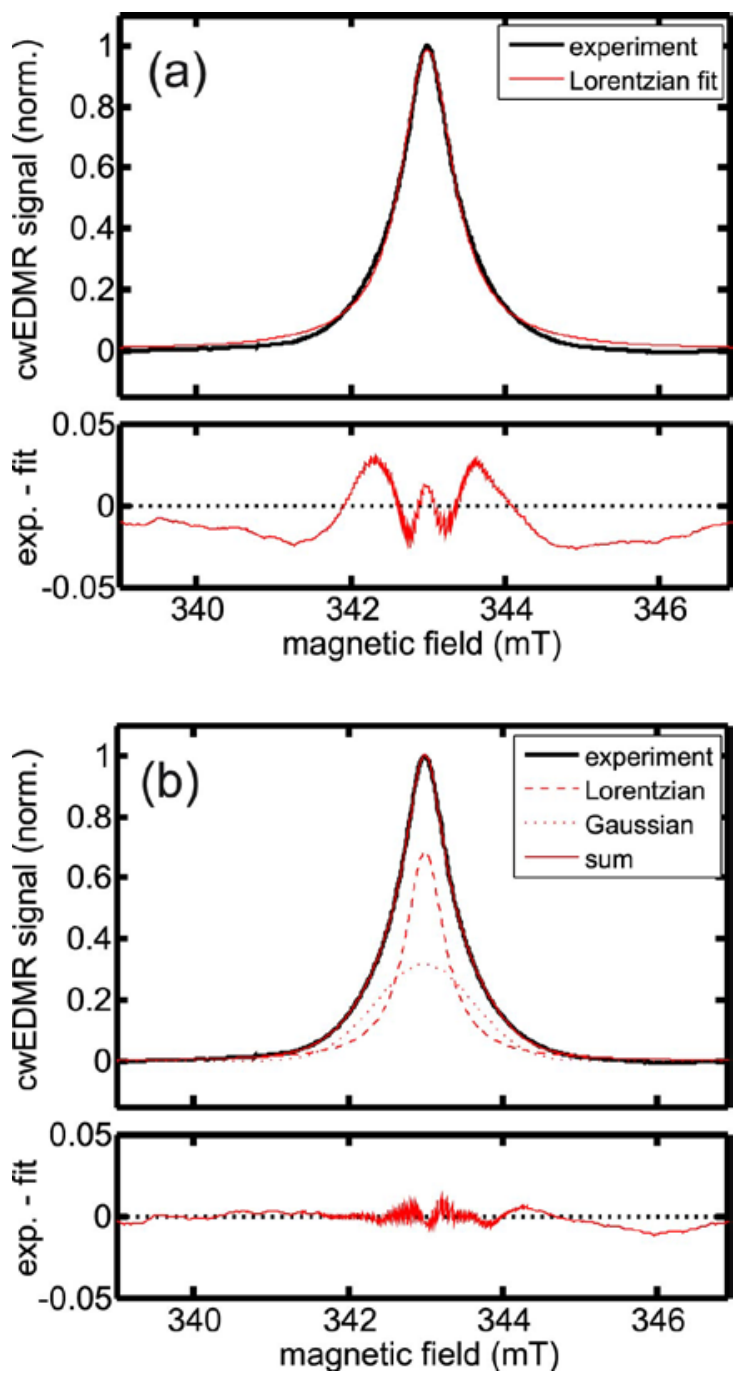

Fig. 14 Integrated cwEDMR spectrum obtained at room temperature without illumination. A fit to experimental data assuming (a) a single resonance with a Lorentzian line shape and (b) two overlapping resonance lines with different line shapes (Lorentzian and Gaussian) are shown 10 along with the experimental spectrum.

In order to investigate transport of $\mathrm{P}^{+}$through the polymer phase, the experimental conditions were chosen accordingly. We used a constant bias voltage of $U=1 \mathrm{~V}$ (resulting current $I=18.7 \mu \mathrm{A}$ ) to electrically inject charge carriers into the blend. Note that this 15 voltage is considerably lower than required for electron injection into MEH-PPV, i.e. lower than the operating voltage in OLEDs made from this material. Further, the large offset between the energy levels of MEH-PPV and PCBM assures that $\mathrm{P}^{+}$reside on the donor polymer and $\mathrm{P}^{-}$on PCBM. ${ }^{142,143}$

20 Fig. 14 a) shows the integrated cwEDMR dark spectrum of the BHJ solar cell obtained employing field modulation and using a constant forward bias for charge carrier injection $(U=1.0 \mathrm{~V}, I=$ $18.7 \mu \mathrm{A})$. The spectrum is similar to cwEDMR spectra of MEHPPV based diodes, ${ }^{140}$ except here we observe a relative increase 25 in the current at resonance. A fit assuming a single Lorentzian line, which is shown alongside with the experimental data in Fig.
14 a, reveals $g=2.0028(3)$. This $g$-value is in accordance with earlier EDMR and light-induced EPR measurements on polarons in PPV and excludes the possibility that PCBM anions ( $g=$ $301.9995)$ significantly contribute to the spectrum. ${ }^{144,}{ }^{145}$ However, the clear deviations between fit and experimental data are much larger than the noise floor and thus indicate that the spectrum consists of several contributions. Fig. 14 b) shows the same experimental spectrum and a two-component fit comprising a 35 narrow Lorentzian line and a broad Gaussian line with indistinguishable $g$-values. The good agreement between the experimental results and the fit corroborates the assumption of a two-component spectrum.

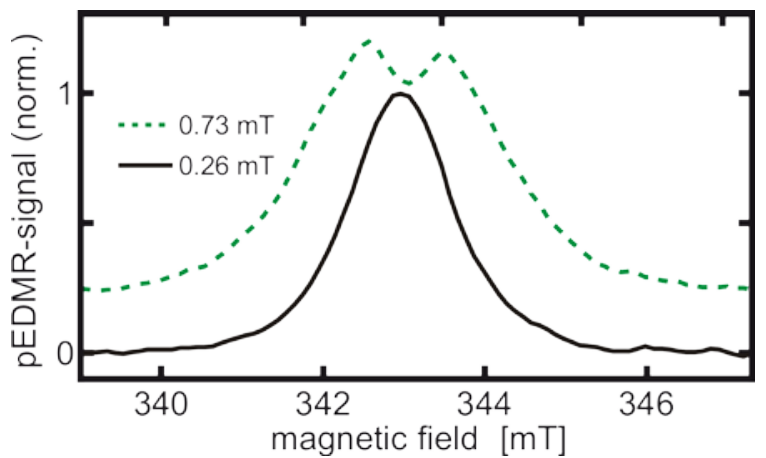

Fig. $15 B_{1}$ dependence of the pEDMR spectrum obtained using a constant $40 \mathrm{mw}$ pulse length of $160 \mathrm{~ns}$.

The cwEDMR line shape shown in Fig. 14 may be explained by two MEH-PPV polaron populations differing in environment and/or mobility. Different environments lead to different $g$ and HFI tensors. Immobile centres then give a Gaussian line, while 45 polaron mobility may average the anisotropies, leading to a pseudo-Voigtian line shape with strong Lorentzian character that was approximated by a purely Lorentzian line in Fig. 14. Yet, the cwEDMR spectrum alone does not allow us to conclude on the microscopic mechanism, i.e. to identify a spin-dependent process 50 involving two separate $S=1 / 2$ species and to exclude scenarios (iii) and (iv) above.

In order to elucidate the microscopic process associated with the EDMR signal we recorded pEDMR spectra for different amplitudes of the magnetic component of the mw field quantified 55 by $B_{1}$. Fig. 15 shows the resulting spectra (integrated current change following a $160 \mathrm{~ns}$ long mw pulse). For low $B_{1}$ we observe a single resonance in accordance with the cwEDMR spectrum in Fig. 14. Upon increasing the mw power this line broadens and a 'dip' appears in the centre of the spectrum, which 60 is particularly pronounced for $B_{1}=0.73 \mathrm{mT}$. This is an indication of coupled spin pairs with overlapping spectra for both constituents of each pair. When $B_{1}$ is sufficiently high to simultaneously excite both spin-pair partners, spin locking occurs. This effect was previously observed for radical pairs in 65 biological systems. ${ }^{146}$ In our case the appearance of spin locking can be rationalized as follows. According to the KSM model, the EDMR spectrum is often assumed to be due to the formation of $S$ $=1 / 2$ spin pairs as part of a transport process in which a rate constant critically depends on the singlet content of the pair. The $70 \mathrm{mw}$ field induces changes to the singlet and triplet populations away from equilibrium, leading to a resonant current change. ${ }^{28}$ In the case of spin locking both spins are manipulated simultaneously, not affecting the singlet content of the pair. Spin locking is most effective in the centre of the spectrum. As a 
result, a pair that is initially in a pure triplet state will not acquire singlet content. ${ }^{147,} 148$ Intersystem crossing (ISC) promotes population transfer between the mixed spin-pair states and thus effectively leads to an increase of the singlet content 5 accompanied by a non-vanishing EDMR signal even in the (unrealistic) case of complete spin locking. Nevertheless, the mwinduced increase of the singlet content is less effective than in the absence of spin locking and thus gives rise to the powerdependent line-shape changes. While the observation of a dip in 10 the centre of the spectrum is persuasive, the spin locking mechanism should also result in a characteristic behaviour in coherent spin nutation experiments (Rabi oscillations).

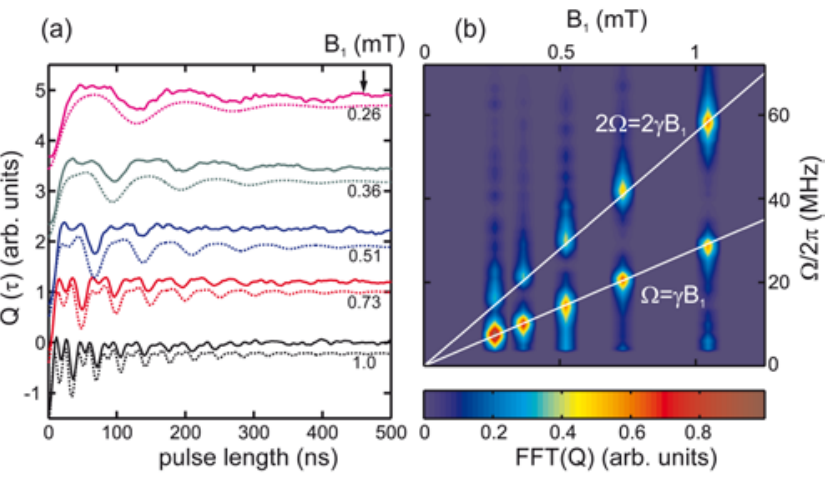

(c)

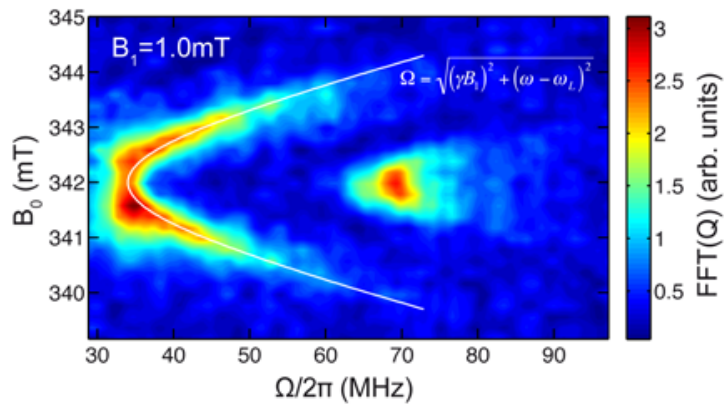

Fig. 16 (a) Electrically detected spin Rabi nutations obtained at room 15 temperature (integrated charge $Q$ as a function of the mw pulse length for several values of $B_{1}$ ). The solid curves show the experimental data (vertically offset for clarity). The dashed curves represent the results of simulations based on the line parameters obtained from the cwEDMR spectrum (Fig. 14) and the mw intensities (see Ref. ${ }^{53}$ for details). (b) Fast 20 Fourier Transform (FFT) of the data shown in (a). (c) FFT of a background-subtracted Rabi nutation measurement $\left(B_{1}=1.0 \mathrm{mT}\right)$ for different amplitudes of the static magnetic field. The solid curve represents the increase in Rabi frequency $\Omega$ as a function of the offset between $B_{0}$ and the resonance field described by the given formula $(\gamma$ : 25 gyromagnetic ratio, $\omega$ : mw frequency, $\omega_{\mathrm{L}}$ : Larmor frequency).

Fig. 16 shows pEDMR-detected Rabi oscillations measured on resonance for different amplitudes of $B_{1}$. At low power $\left(B_{1}=\right.$ $0.26 \mathrm{mT})$ the frequency of the oscillation $(\Omega / 2 \pi)$ corresponds to the Rabi frequency, $\Omega=\gamma B_{1}$, where $\gamma$ is the gyromagnetic ratio, 30 which here is found to take the value expected for a total spin $1 / 2$. Upon increasing $B_{1}$ the Rabi frequency increases and a second frequency component at $2 \Omega$ is observed. This component rises in intensity when increasing $B_{1}$, whereas the $\Omega$ component decreases. The power level at which the second frequency 35 component appears ( $\sim 0.36 \mathrm{mT})$ approximately coincides with the change of the pEDMR spectrum shown in Fig. 14. These observations unambiguously illustrate that spin locking of (initially) weakly coupled pairs of $S=1 / 2$ particles is occurring and that these are responsible for the spin-dependent process 40 associated with the EDMR spectrum.

Fig. 16(c) illustrates the influence of the static magnetic field on the Rabi frequency for $B_{1}=1.0 \mathrm{mT}$. The $\Omega$ - as well as the $2 \Omega$ component can clearly be observed in this plot. The characteristic shape of the $\Omega$-component can be rationalized by the formula 45 shown in Fig. 16 (c). The off-resonance term $\omega$ - $\omega_{\mathrm{L}}$ causes the Rabi frequency to increase with the field offset when $B_{0}$ deviates from the resonance-field position.

In the absence of spin locking the mw field directly affects the singlet content of the pairs at the Rabi frequency $\Omega$ (cf. Sec. 2). 50 Spin locking decouples the singlet state from the triplet manifold, so that the population transfer is restricted to be within the triplet states (which are not necessarily eigenstates). These changes occur at $2 \Omega$. In the presence of ISC the singlet and triplet populations tend to equalize. ISC may, e.g., be caused by 55 hyperfine interaction between polaron and nuclear spins. The link for population transfer between $\mathrm{S}$ and $\mathrm{T}^{0}$ is thus partly reestablished. However, in this case the singlet content of the spinpair oscillates at $2 \Omega$, since the $\mathrm{T}^{0}$ state content is varying at this frequency. These changes manifest in the spin-dependent current 60 changes.

The fact that we observe spin locking establishes the assumption that the EDMR spectrum is caused by weakly coupled pairs of $\mathrm{S}$ $=1 / 2$ particles, leaving two possibilities for the underlying microscopic mechanism, namely spin-dependent bipolaron 65 formation from $\mathrm{P}^{+} \mathrm{P}^{+}$precursor pairs and $\mathrm{P}^{+} \mathrm{P}^{-}$recombination. Distinguishing between these mechanisms requires careful consideration of the experimental conditions and the device structure, because the experimental observation alone is fully consistent with either a unipolar $\mathrm{P}^{+} \mathrm{P}^{+}$(bipolaron formation) or a ${ }_{70} \mathrm{P}^{+} \mathrm{P}^{-}$(recombination) process. Since the discrimination between both mechanisms is of crucial importance, and recent pEDMR measurements performed on pure MEH-PPV devices at cryogenic temperatures revealed EDMR signals due to $\mathrm{P}^{+} \mathrm{P}^{-}$ recombination, ${ }^{141}$ we provide a detailed description of how we 75 arrive at our data interpretation.

We deduce that in MEH-PPV:PCBM blend devices operated at $U$ $=1 \mathrm{~V}$ the pEDMR is due to the unipolar $\mathrm{P}^{+} \mathrm{P}^{+}$process. This is primarily based on the absence of a resonance at $g=1.9995$, the PCBM radical anion $g$-value. A plausible cause for the absence of $80 \mathrm{PCBM} \mathrm{P}^{-}$resonance at room temperature would be that the line is too broad to be detected due to the increased spin-relaxation rate. However, spin locking requires the simultaneous excitation of significant portions of both spin partner resonances. This cannot be achieved for a resonance that is too broad to be 85 resolved in the PEDMR spectrum at the mw powers used.

$\mathrm{P}^{+} \mathrm{P}^{-}$recombination on $\mathrm{MEH}-\mathrm{PPV}$ could be compatible with the EDMR data, and may explain pEDMR and ODMR studies on pristine MEH-PPV films. ${ }^{54}$ However, it is not a credible explanation for blends. We exclude $\mathrm{P}^{+} \mathrm{P}^{-}$recombination on $\mathrm{MEH}-$ 90 PPV because of the low probability for electron injection into MEH-PPV in the blend at $U=1 \mathrm{~V}$. Even if negative polarons formed on the polymer, the presence of PCBM, which has a large offset in its HOMO and LUMO levels as compared to $\mathrm{MEH}$ $\mathrm{PPV},{ }^{142,143}$ would result in negative charge carriers on the PCBM 95 and positive charge carriers on the MEH-PPV in the blend. ${ }^{149}$

Thus, we are left to conclude that the most reasonable explanation for the spin locking signal we observe is spin- 
dependent bipolaron formation on MEH-PPV. We infer from Fig. 14 that two different $\mathrm{P}^{+}$entities are detected and are relevant to the transport mechanism, indicating the model in which the spindependent positive bipolaron formation involves partners with 5 dissimilar environments and/or mobilities and, accordingly, different line shapes. A mechanism consistent with this model is trap-mediated isoenergetic hopping. Deep level transient spectroscopy detects a hole trap 0.3-0.4 eV above the HOMO. ${ }^{150}$

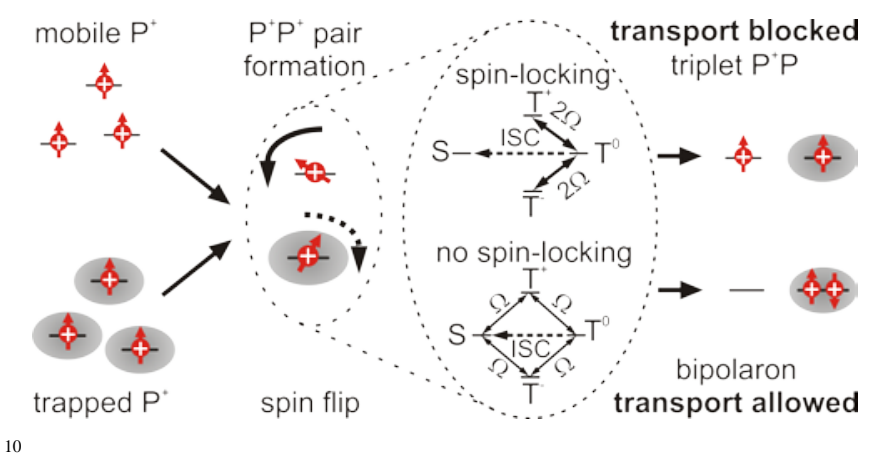

Fig. 17 Bipolaron formation under resonant mw excitation. In the scheme polaron charge and spin are indicated by + and arrow, respectively. Grey shaded ellipses indicate trap sites accommodating $\mathrm{P}^{+}$or bipolarons. From left to right polarons form weakly coupled spin pairs in a mixed S T state 15 upon encounter of mobile and trapped polarons. Resonant mw excitation alters the $\mathrm{S}$ and $\mathrm{T}$ content, with an oscillation frequency $\Omega$ (no spin locking) or $2 \Omega$ (spin locking). ISC transfers population from $\mathrm{T}^{0}$ to $\mathrm{S}$. Depending on the $\mathrm{S}$ and $\mathrm{T}$ content of the pair, either triplet $\mathrm{P}^{+} \mathrm{P}^{+}$pairs (transport blocked) or bipolarons (transport allowed) are formed.

20 If the energy of the mobile $\mathrm{P}^{+}$and the doubly occupied bipolaron state is similar, isoenergetic hopping can occur. The bipolaron formation process in accordance with our EDMR results is shown in Fig. 17.

Mobile $\mathrm{P}^{+}$drift in the electric field provided by the bias voltage. ${ }_{25}$ When a mobile $\mathrm{P}^{+}$encounters a trapped $\mathrm{P}^{+}$, a $\mathrm{P}^{+} \mathrm{P}^{+}$pair forms. Depending on the relative spin orientation of both charge carriers, the mobile species may or may not hop to the site of the trapped $\mathrm{P}^{+}$to form a bipolaron. Since the hopping process occurs predominantly along the direction of the electric field, it results in 30 a current enhancement and consequently yields a positive EDMR signal. ${ }^{86}$ It is conceivable that the bipolaron is stabilized by a negative charge on an adjacent PCBM molecule. ${ }^{151}$

The process impacts solar cell operation: after optical generation of an exciton and subsequent separation into $\mathrm{P}^{+}$and $\mathrm{P}^{-}$, the ${ }_{35}$ positive polaron is transported towards the ITO electrode. If another (trapped) $\mathrm{P}^{+}$with parallel spin orientation is encountered, the transport path involving positive bipolaron formation is blocked, so hindering charge transport to the electrode, and hence lowering the efficiency of the solar cell.

40 This example shows that pEDMR can successfully identify loss pathways relevant to carrier collection in organic solar cells under ambient conditions. The underlying mechanisms are not accessible by cwEDMR or EPR techniques; however, a profound understanding of charge transport on a molecular level is a 45 necessary prerequisite for prospective solar cell concepts utilizing the properties of the charge-carrier spins.

It is expected that pEDMR will contribute to the progress in a number of disciplines dealing with transport in organic semiconductors due to the importance of point defects for their 50 electrical properties. ${ }^{152}$

A particular research area exemplifying the lack of knowledge regarding microscopic details of transport is the field of organic magnetoresistance, i.e. the change in resistivity of an organic semiconductor film as a function of an external magnetic field. ${ }_{55}$ Several microscopic models, all of them involving localized (paramagnetic) states, were put forward to explain this effect. ${ }^{153}$ It seems that depending on the experimental conditions different mechanisms prevail, but discriminating between them solely based on magnetoresistance measurements is impossible. EDMR ${ }_{60}$ has the potential to clarify this important issue because the different processes are expected to result in dissimilar EDMR signals. However, no combined magnetoresistance / EDMR study on organic semiconductors has been reported yet.

Another aspect that could be elucidated with the aid of pEDMR is 65 the investigation of intermediate states involved in charge separation following exciton fission at the donor:acceptor interface in OSC. The exact role of these states dubbed charge transfer states in the course of free charge-carrier generation is subject of intense debate. ${ }^{154-156}$ This can partly be attributed to the 70 fact that it is often not clear whether the species probed by the respective experimental technique (mostly time-resolved optical spectroscopy) indeed represent the relevant species involved in free charge-carrier generation. In many cases it is impossible to exclude that the signals originate from trapped charge carriers not 75 contributing to the photocurrent of the OSC. pEDMR is a suitable technique for studying the influence of charge transfer complexes on the free charge carrier yield as it is inherently only sensitive to current-influencing processes.

\section{Conclusion}

${ }_{80}$ In the previous sections we demonstrated the potential of advanced pEDMR detection schemes to study spin-dependent transport processes in fully processed solar cells. Strategies were presented to study defect structures in TFS and OPV solar cells on the micro scale, ${ }^{50}$ locate defect centres in multi layer devices ${ }^{48}$, ${ }_{85}{ }^{53}$ and conclude on the underlying transport pathways. ${ }^{32,48,53}$

For thin film $\mu \mathrm{c}-\mathrm{Si}: \mathrm{H}$ pin solar cells we could show that electron hopping is the dominating spin-dependent transport pathway in a$\mathrm{Si}: \mathrm{H}$ as well as in the $\mu \mathrm{c}-\mathrm{Si}: \mathrm{H}$ absorber layer at low temperatures. In both materials hopping involves conduction band-tail states. 90 However, while in a-Si:H hopping occurs between two different paramagnetic states, namely conduction band-tail states and phosphorus donor states, it happens solely via conduction bandtail states in the $\mu \mathrm{c}-\mathrm{Si}: \mathrm{H}$ layer. We found that conduction bandtail states in $\mu \mathrm{c}-\mathrm{Si}: \mathrm{H}$ are located in a material phase that is ${ }_{95}$ depleted from hydrogen, which is only the case for the crystalline contribution in $\mu \mathrm{c}-\mathrm{Si}: \mathrm{H}$. Thereby we were able to assign the $g_{\mathrm{CE}}$ EDMR signal to twin boundaries inside crystalline grains. Such states can act as trapping sites for mobile charge carriers and hence will limit the solar cell efficiency.

100 In the dark current at room temperature we observe a so far unidentified signal with the signature of a dangling bond in intrinsic $\mu \mathrm{c}-\mathrm{Si}: \mathrm{H}$. We could clearly show that this signal is not associated with a direct capture process since the typical features of spin locking that is expected for this process are missing. The 105 underlying spin dependent transport process is not clear yet.

In the organic MEH-PPV/PCBM solar cells we observed a process which strongly resembles charge-carrier hopping in TFS materials, namely bipolaron formation from positively charged MEH-PPV polarons. This process involves $\mathrm{P}^{+}$pairs, where the 110 partners differ in their mobility and local environment. We found 
that when mobile and trapped $\mathrm{P}^{+}$encounter, a bipolaron may be formed when the polaron spins are aligned anti parallel to each other. In the alternative case this transport channel is blocked.

Despite the dissimilar material composition, morphology and 5 device architecture of TFS and OPV solar cells under study, we found that similar spin-dependent transport processes impact their operation. However, depending on the operation conditions (temperature, bias voltage) and EDMR resonance fields other spin-dependent transport processes like recombination may 10 dominate spin-dependent transport. In TFS, in particular a-Si:H, as well as in OPV materials EDMR studies yielded clear evidence for spin-dependent recombination.

We could show that unique information to distinguish between spin-dependent transport processes may be obtained by the 15 combination of different advanced pEDMR methods. For TFS as well as OPV systems detailed understanding of spin-dependent transport mechanisms and the accompanied paramagnetic states in the bulk material, at grain boundaries and interfaces is required in the quest for optimized solar cells.

\section{${ }_{20}$ 7. Outlook}

The capabilities of advanced pEDMR techniques are certainly not limited to the material systems presented here, but will be also useful for the characterization of future solar cell designs and other electronic devices. As these devices will include multiple

25 layers of varying morphology and material composition, complex contact and interface structures as well as nanostructures embedded in organic and inorganic matrices, dedicated pEDMR approaches have to be developed to obtain structural and functional details at different length scales and time intervals. The 30 use of advanced pEDMR spectroscopy for solar cell research only started and currently a rapidly growing arsenal of pEDMR based methods and its multi-resonance extensions is being developed. In the following we identify major fields, where recent progress in EDMR methodology as well as novel 35 theoretical approaches may significantly impact pEDMR based solar cell research.

A) Novel pulse sequences

Recent years witnessed the development of an increasing number of pEDMR detection schemes. The major benefits of these 40 techniques are strongly increased spectral and temporal resolution. The possibility to access pEDMR signals in the time domain permits studies in light induced charge transfer processes in PV materials on the ns to ms time scale. Thereby short lived transient or metastable states may be investigated by optical ${ }_{45}$ pump - pEDMR probe experiments. High spectral resolution on the other hand is a prerequisite for the precise determination of spin coupling parameters. In particular $\mathrm{g}$ and A tensors contain important information on the wave function and the atomic structure around paramagnetic defects and localized charge 50 transfer in inorganic as well as in organic materials.

B) Multifrequency $p E D M R$

The availability of pulsed multi-frequency (MF) EPR spectrometers up to several hundred $\mathrm{GHz}$ paved the way for pEDMR experiments at different resonance frequencies. ${ }^{75,98,157}$

55 Thereby the information content extractable from advanced pEDMR methods can be even increased.

Following the pathway of modern MF EPR spectroscopy ${ }^{158}$ pEDMR experiments carried out at different frequency bands are capable of further increasing the spectral resolution. Thereby 60 spectrally overlapping paramagnetic sites with slightly different $g$ values (see e.g. Fig. 8) as well as canonical g tensor components may be resolved by increasing the external magnetic field. This allows for an unambiguous identification of paramagnetic states contributing to the same EDMR spectrum. In addition, orientation ${ }_{65}$ selective multi-resonance experiments like EDESEEM and EDENDOR may be performed to obtain the relative orientation of $g$ and A tensors. However, electrical detection provides the potential to go even beyond the limits of MF EPR. Due to its high detection sensitivity EDMR experiments can be performed over a 70 very wide field range on the same sample size, ranging from $B_{0}<$ $10 \mathrm{G}^{157}$ to $B_{0}>80 \mathrm{kG}^{75}$ By changing the resonance condition from the low field limit up to very high resonance fields the spin transition probabilities and populations are drastically changed, which strongly changes the spectral shape and may induce ${ }_{75}$ switches between different spin-dependent processes. ${ }^{75}$, 98, 157 Thereby significantly increased information about the spindependent transport mechanisms and the involved paramagnetic sites can be obtained, which may help to study spin-dependent transport processes at a level hardly achievable with any other 80 method.

C) Combination with quantum chemical approaches

Detailed structural models as well as the location of paramagnetic states in the solar cell band structure may be obtained by combining pEDMR spectroscopy with density functional theory

85 (DFT). Despite the wealth of information that is contained in the spin coupling parameters a direct relation between these parameters and the particular structural details is oftentimes not possible. This situation can be improved by simultaneous DFT calculations of the spin coupling parameters on computer 90 generated model structures. DFT codes capable of calculating g and A tensors in crystalline $e^{159-161}$ and non crystalline ${ }^{162} \mathrm{Si}$ materials became available only recently. First applications on $\mathrm{Si} / \mathrm{SiO} 2$ interfaces ${ }^{37}$ proved that by combining DFT calculations with the sensitivity and spectral resolution of advanced pEDMR 95 detailed structural information of recombination active defects at Si interfaces may be obtained. In organic chemistry the calculation of spin coupling parameters is widely used to study cofactor radicals in proteins. ${ }^{163}$ However, the application of this powerful approach to function determining paramagnetic sites in 100 organic solar cell has only started. ${ }^{164}$

\section{D) Spatially resolved pEDMR}

High resolution pEDMR methods in combination with quantum chemical approaches primarily target at the local structure on the nanoscale. Due to its ultimate sensitivity pEDMR provides the 105 possibility to carry out spatially resolved experiments with $\mu \mathrm{m}$ mm resolution. pEDMR microscopy would provide additional valuable information about the distribution of defects at interfaces, grain boundaries and in the bulk. Since they require tailor made sample probes, mw excitation schemes and magnet 110 equipment such experiments are very demanding. Nevertheless, several work groups met this challenge and are currently evaluating the feasibility of such spatially resolved pEDMR experiments e.g. at TU Munich and Technion Haifa.

E) Device simulations including spin-dependent transport 115 processes

pEDMR is a reliable technique to identify paramagnetic states involved in spin-dependent electronic processes in thin-film 
silicon solar cells. However, it is not a priori clear whether the observed electronic process (e.g. charge carrier recombination at deep defects) is a performance dominating contribution or a minor part of the overall current, since pEDMR is merely 5 sensitive to spin-dependent processes. In order to relate the observed spin-dependent processes to global charge carrier transport processes of solar cells, pEDMR measurements have to be complemented by device simulations. Several studies reported analytic or numerical device simulation, which include the spin 10 dependence by explicitly simulating singlet and triplet populations of paramagnetic states (e.g. deep defects) or by introducing a perturbative change of the capture cross section. ${ }^{12}$, 91, 128 By comparing a joint model of the current-voltage characteristics and the relative EDMR signal with the actual 15 experimental values can decide if the observed electronic process is indicative of a transport channel important for device operation. A modelling of the EDMR signal intensities, resonance fields and absolute signs was successfully achieved in a-Si:H solar cells using a semiclassical model based on rate equations ${ }^{91}$ 20 and in $\mu \mathrm{c}$-Si solar cells by either simulating a two-diode model ${ }^{12}$ or a numerical model ${ }^{128}$. In all the above cases the current-voltage characteristics and the EDMR signal could be modelled quantitatively assuming a single spin-dependent recombination process which indicates that EDMR monitors the most important 25 electronic processes of solar cells, albeit the typically small magnitude of mw induced current change. However, further refinement is needed to model pEDMR signals occurring at cryogenic temperatures and advanced quantum chemical calculations are needed for a theoretical prediction of rate 30 coefficients to approach the goal of parameter-free simulations of EDMR signals.

A combination of these techniques can yield a multiscale picture of charge transport and loss mechanisms in fully processed solar cells at ambient as well as at cryogenic temperatures. It is our 35 belief that among the numerous spectroscopic techniques, that are used to characterize and understand solar cells, pEDMR will play an important role in the endeavour to understand dominant factors that determine charge transport and loss mechanisms.

\section{Acknowledgement}

${ }_{40}$ We are thankful to University of St. Andrews and FZ Jülich for providing MeH-PPV:PCBM and $\mu \mathrm{c}-\mathrm{Si}: \mathrm{H}$ pin solar cells, respectively. Stimulating discussions with D. Keeble (Uni Dundee), I. Samuel (St. Andrews), F. Finger, O. Astakhov (both FZ Jülich) and B. Rech (HZB) are gratefully acknowledged. The 45 work was partially funded by the German Ministry of Education and Research within the network project EPR-Solar (Grant No. 03SF0328).

\section{Notes and references}

${ }^{a}$ Helmholtz-Zentrum Berlin für Materialien und Energie, Institute for 50 Silicon Photovoltaics, Kekulestrasse 5, 12489 Berlin, Germany,

*E-mail: alexander.schnegg@helmholtz-berlin.de

Fax: ++49 (0)30 806241333; Tel: ++49(0)30 806241373;

**E-Mail: lips@helmholtz-berlin.de

Fax: ++49 (0)30 806241333; Tel: ++49 (0)30 806241353;

$55{ }^{b}$ Free University Berlin, Institute for Experimental Physics, Arnimalle 14, 14195 Berlin,Germany

1. A. Cho, Science, 2010, 329, 1284-1284.

2. R. A. Kerr, Science, 2010, 329, 780-781.
60 3. M. A. Green, K. Emery, Y. Hishikawa, W. Warta and E. D. Dunlop, Prog. Photovol.: Res. Appl., 2012, 20, 12-20.

4. A. Shah, P. Torres, R. Tscharner, N. Wyrsch and H. Keppner, Science, 1999, 285, 692-698.

5. A. G. Aberle, Thin Solid Films, 2009, 517, 4706-4710.

65 6. T. Unold and H. W. Schock, Annu. Rev. Mater. Sci., 2011, 41, 297321.

7. B. E. Hardin, H. J. Snaith and M. D. McGehee, Nature Phot., 2012, 6, 162-169.

8. C. J. Brabec, N. S. Sariciftci and J. C. Hummelen, Adv. Funct. 70 Mater., 2001, 11, 15-26.

9. M. A. Green, K. Emery, Y. Hishikawa and W. Warta, Prog. Photovol.: Res. Appl., 2009, 17, 320-326.

10. W. Shockley and H. J. Queisser, J. Appl. Phys., 1961, 32, 510-519.

11. P. K. Nayak, G. Garcia-Belmonte, A. Kahn, J. Bisquert and D. 75 Cahen, Energy Environ. Sci., 2012, 5, 6022-6039.

12. K. Lips, C. Boehme and W. Fuhs, IEE Proc. - Circ. Dev. Syst., 2003, 150, 309-315.

13. A. Honig, Phys. Rev. Lett., 1966, 17, 186-188.

14. R. Maxwell and A. Honig, Phys. Rev. Lett., 1966, 17, 188-190.

80 15. J. Schmidt and I. Solomon, Compt. Rend. Acad. Sci. B, 1966, 263, 169-172.

16. M. Xiao, I. Martin, E. Yablonovitch and H. W. Jiang, Nature, 2004, 430, 435-439.

17. D. R. McCamey, H. Huebl, M. S. Brandt, W. D. Hutchison, J. C. 85 McCallum, R. G. Clark and A. R. Hamilton, Appl. Phys. Lett., 2006, 89, 182115.

18. F. H. L. Koppens, K. C. Nowack and L. M. K. Vandersypen, Phys. Rev. Lett., 2008, 100.

19. K. Lips and W. Fuhs, J. Appl. Phys., 1993, 74, 3993-3999.

90 20. M. Stutzmann, M. S. Brandt and M. W. Bayerl, J. Non-Cryst. Solids, 2000, 266-269, 1-22.

21. K. Lips, P. Kanschat and W. Fuhs, Sol. Energy Mat. Sol. Cells, 2003, 78, 513-541.

22. R. Mueller, P. Kanschat, S. von Aichberger, K. Lips and W. Fuhs, J.

$95 \quad$ Non-Cryst. Solids, 2000, 266-269, 1124-1128.

23. P. Kanschat, K. Lips and W. Fuhs, J. Non-Cryst. Solids, 2000, 266, 524-528.

24. V. Lang, J. D. Murphy, R. J. Falster and J. J. L. Morton, J. Appl. Phys., 2012, 111, 013710-013717.

100 25. C. Boehme and K. Lips, Appl. Phys. Lett., 2001, 79, 4363-4365.

26. C. Boehme and K. Lips, Phys. Rev. Lett., 2003, 91, 246603.

27. A. Schweiger and G. Jeschke, Principles of pulse electron paramagnetic resonance, Oxford University Press, Oxford, UK ; New York, 2001.

105 28. C. Boehme and K. Lips, Phys. Rev. B, 2003, 68, 245105.

29. K. Lips and C. Boehme, J. Mater. Sci.: Mater. Electron., 2003, 14, 635-639.

30. C. Boehme and K. Lips, Physica B, 2006, 376, 930-935.

31. A. R. Stegner, C. Boehme, H. Huebl, M. Stutzmann, K. Lips and M. 110 S. Brandt, Nature Phys., 2006, 2, 835-838.

32. J. Behrends, K. Lips and C. Boehme, Phys. Rev. B, 2009, 80, 045207.

33. D. R. McCamey, J. Van Tol, G. W. Morley and C. Boehme, Science, 2010, 330, 1652-1656.

34. H. Huebl, F. Hoehne, B. Grolik, A. R. Stegner, M. Stutzmann and M. 115 S. Brandt, Phys. Rev. Lett., 2008, 100, 177602.

35. F. Hoehne, H. Huebl, B. Galler, M. Stutzmann and M. S. Brandt, Phys. Rev. Lett., 2010, 104 
36. F. Hoehne, L. Dreher, H. Huebl, M. Stutzmann and M. S. Brandt, Phys. Rev. Lett., 2011, 106.

37. F. Hoehne, J. M. Lu, A. R. Stegner, M. Stutzmann, M. S. Brandt, M. Rohrmuller, W. G. Schmidt and U. Gerstmann, Phys. Rev. Lett., 2011, 106

38. C. Boehme, P. Kanschat and K. Lips, J. Non-Cryst. Solids, 2002, 299, 566-570.

39. C. Boehme, P. Kanschat and K. Lips, Nucl. Instr. Meth. B, 2002, 186, 30-35.

10 40. C. Boehme and K. Lips, Appl. Magn. Res., 2004, 27, 109-122.

41. C. Boehme and K. Lips, phys. stat. sol. (c), 2004, 1, 1255-1274.

42. C. Boehme and K. Lips, J. Non-Cryst. Solids, 2004, 338-40, 434-439.

43. K. Lips, C. Boehme and W. Fuhs, J. Non-Cryst. Solids, 2004, 338-40, 702-705.

15 44. C. Boehme, F. Friedrich, T. Ehara and K. Lips, Thin Solid Films, 2005, 487, 132-136.

45. T. W. Herring, S. Y. Lee, D. R. McCamey, P. C. Taylor, K. Lips, J. Hu, F. Zhu, A. Madan and C. Boehme, Phys. Rev. B, 2009, 79, 195205.

20 46. F. Friedrich, C. Boehme and K. Lips, J. Appl. Phys., 2005, 97, -.

47. C. Boehme, J. Behrends, K. von Maydell, M. Schmidt and K. Lips, J. Non-Cryst. Solids, 2006, 352, 1113-1116.

48. J. Behrends, A. Schnegg, C. Boehme, S. Haas, H. Stiebig, F. Finger, B. Rech and K. Lips, J. Non-Cryst. Solids, 2008, 354, 2411-

25 2415.

49. J. Behrends, A. Schnegg, M. Fehr, A. Lambertz, S. Haas, F. Finger, B. Rech and K. Lips, Philos. Mag., 2009, 89, 2655 - 2676.

50. M. Fehr, J. Behrends, S. Haas, B. Rech, K. Lips and A. Schnegg, Phys. Rev. B, 2011, 84, 193202.

30 51. W. Harneit, C. Boehme, S. Schaefer, K. Huebener, K. Fostiropoulos and K. Lips, Phys. Rev. Lett., 2007, 98, 216601.

52. J. M. Lupton and C. Boehme, Nature Mater., 2008, 7, 598-598.

53. J. Behrends, A. Schnegg, K. Lips, E. A. Thomsen, A. K. Pandey, I. D. W. Samuel and D. J. Keeble, Phys. Rev. Lett., 2010, 105.

35 54. S. Y. Lee, S. Y. Paik, D. R. McCamey, J. Yu, P. L. Burn, J. M. Lupton and C. Boehme, J. Am. Chem. Soc., 2011, 133, 20192021.

55. S. Schaefer, S. Saremi, J. Behrends, K. Fostiropoulos, K. Lips and W. Harneit, phys. stat. sol. (b), 2009, 246, 2844-2848.

40 56. H. Mell and J. Stuke, J. Non-Cryst. Solids, 1970, 4, 304-310.

57. B. Movaghar and L. Schweitzer, J. Phys. C, 1978, 11, 125-135.

58. A. Kurobe and H. Kamimura, J. Non-Cryst. Solids, 1983, 59-60, 4144.

59. T. L. Francis, O. Mermer, G. Veeraraghavan and M. Wohlgenannt, $45 \quad$ New. J. Phys., 2004, 6, 1-8.

60. F. J. Wang, H. Bassler and Z. V. Vardeny, Phys. Rev. Lett., 2008, 101, 236805.

61. B. Hu and Y. Wu, Nature Mater., 2007, 6, 985-991.

62. N. M. Atherton, Principles of electron spin resonance, Ellis Horwood

$50 \quad$ : PTR Prentice Hall, New York, 1993.

63. C. D. Buckley, D. A. Hunter, P. J. Hore and K. A. Mclauchlan, Chem. Phys. Lett., 1987, 135, 307-312.

64. A. J. Hoff, Advanced EPR : applications in biology and biochemistry, Elsevier, Amsterdam ; New York, NY, U.S.A.,

55 1989.

65. D. J. Lepine, Phys. Rev. B, 1972, 6, 436-441.
66. N. Kishimoto, K. Morigaki and K. Murakami, J. Phys. Soc. Jpn., 1981, 50, 1970-1977.

67. I. Solomon, Solid State Commun., 1976, 20, 215-217.

60 68. E. A. Schiff, AIP Conf. Proc., 1981, 73, 233-237.

69. R. A. Street, Philos. Mag. B, 1982, 46, 273-278.

70. F. C. Rong, G. J. Gerardi, W. R. Buchwald, E. H. Poindexter, M. T. Umlor, D. J. Keeble and W. L. Warren, Appl. Phys. Lett., 1992, 60, 610-612.

65 71. L. S. Swanson, J. Shinar, A. R. Brown, D. D. C. Bradley, R. H. Friend, P. L. Burn, A. Kraft and A. B. Holmes, Phys. Rev. B, 1992, 46, 15072-15077.

72. V. Dyakonov, N. Gauss, G. Rosler, S. Karg and W. Riess, Chem. Phys., 1994, 189, 687-695.

70 73. R. N. Ghosh and R. H. Silsbee, Phys. Rev. B, 1992, 46, 12508-12525.

74. D. D. Thornton and A. Honig, Phys. Rev. Lett., 1973, 30, 909-912.

75. G. W. Morley, D. R. McCamey, H. A. Seipel, L. C. Brunel, J. van Tol and C. Boehme, Phys. Rev. Lett., 2008, 101, 207602.

76. F. Neppl, J. P. Kotthaus and J. F. Koch, Phys. Rev. B, 1979, 19, 75 5240-5250.

77. C. Lerner, K. Lips and W. Fuhs, J. Non-Cryst. Solids, 1998, 230, 1177-1181.

78. K. Lips, M. Block, W. Fuhs and C. Lerner, J. Non-Cryst. Solids, 1993, 166, 697-700.

80 79. D. Kaplan, I. Solomon and N. F. Mott, Journ. de Phys. Lettr., 1978, 39, L51-L54.

80. C. Boehme and K. Lips, in Charge transport in disordered solids with applications in electronics, ed. S. Baranovski, Wiley, Chichester, England ; Hoboken, NJ, 2006, pp. 179-219.

85 81. R. A. Street, Hydrogenated amorphous silicon, Cambridge University Press, Cambridge ; New York, 1991.

82. A. J. Heeger, N. S. Sariciftci and E. B. Namdas, Semiconducting and metallic polymers, Oxford University Press, 2010.

83. H. Kamimura and $\mathrm{H}$. Aoki, The physics of interacting electrons in $90 \quad$ disordered systems, Clarendon Press; Oxford University Press, Oxford, New York, 1989.

84. D. Ephron, M. R. Beasley, H. Bahlouli and K. A. Matveev, Phys. Rev. B, 1994, 49, 2989-2992.

85. J. Stuke, Ann. Rev. Mat. Sci., 1985, 15, 79-102.

95 86. P. A. Bobbert, T. D. Nguyen, F. W. A. van Oost, B. Koopmans and M. Wohlgenannt, Phys. Rev. Lett., 2007, 99, 216801.

87. J. Behrends, PhD thesis, Freie Universität Berlin, 2009.

88. B. Movaghar, B. Ries and L. Schweitzer, Philos. Mag. B, 1980, 41, 159-167.

100 89. F. C. Rong, W. R. Buchwald, E. H. Poindexter, W. L. Warren and D. J. Keeble, Solid-State Electron., 1991, 34, 835-841.

90. Z. Xiong and D. J. Miller, Appl. Phys. Lett., 1993, 63, 352-354.

91. K. Lips, C. Lerner and W. Fuhs, J. Non-Cryst. Solids, 1996, 200, 267-270.

105 92. A. V. Barabanov, V. A. Lvov and O. V. Tretyak, phys. stat. sol. (b), 1998, 207, 419-427.

93. V. Rajevac, C. Boehme, C. Michel, A. Gliesche, K. Lips, S. D. Baranovskii and P. Thomas, Phys. Rev. B, 2006, 74, 245206.

94. A. Gliesche, C. Michel, V. Rajevac, K. Lips, S. D. Baranovskii, F. $110 \quad$ Gebhard and C. Boehme, Phys. Rev. B, 2008, 77, 245206.

95. D. R. McCamey, H. A. Seipel, S. Y. Paik, M. J. Walter, N. J. Borys, J. M. Lupton and C. Boehme, Nature Mater., 2008, 7, 723728. 
96. D. R. McCamey, G. W. Morley, H. A. Seipel, L. C. Brunel, J. van Tol and C. Boehme, Phys. Rev. B, 2008, 78, 045303.

97. W. Harneit, C. Boehme, S. Schaefer, K. Huebener, K. Fostiropoulos and K. Lips, Phys. Rev. Lett., 2008, 100, -.

5 98. C. C. Lo, V. Lang, R. E. George, J. J. L. Morton, A. M. Tyryshkin, S. A. Lyon, J. Bokor and T. Schenkel, Phys. Rev. Lett., 2011, 106.

99. C. Boehme, Philipps Universitaet Marburg, 2002.

100.B. M. Tadjikov, A. V. Astashkin and Y. Sakaguchi, Chem. Phys. $10 \quad$ Lett., 1998, 283, 179-186.

101.F. Hoehne, L. Dreher, J. Behrends, M. Fehr, H. Huebl, K. Lips, A. Schnegg, M. Suckert, M. Stutzmann and M. S. Brandt, Rev. Sci. Instr., 2012, 83.

102.E. R. Davies, Phys. Lett. A, 1974, 47, 1-2.

15 103.L. Dreher, F. Hoehne, M. Stutzmann and M. S. Brandt, Phys. Rev. Lett., 2012, 108, 027602.

104.S. Haas, A. Gordijn and H. Stiebig, Prog. Photovol.: Res. Appl., 2008, 16, 195-203.

105.D. E. Carlson and C. R. Wronski, Appl. Phys. Lett., 1976, 28, 67120673.

106.B. Rech, T. Roschek, T. Repmann, J. Mueller, R. Schmitz and W. Appenzeller, Thin Solid Films, 2003, 427, 157-165.

107.M. Tzolov, F. Finger, R. Carius and P. Hapke, J. Appl. Phys., 1997, 81, 7376-7385.

25 108.A. Shah, J. Meier, E. Vallat-Sauvain, N. Wyrsch, U. Kroll, C. Droz and U. Graf, Sol. Energy Mat. Sol. Cells, 2003, 78, 469-491.

109.F. Finger, C. Malten, P. Hapke, R. Carius, R. Fluckiger and H. Wagner, Phil. Mag. Lett., 1994, 70, 247-254.

110.J. Mueller, F. Finger, R. Carius and H. Wagner, Phys. Rev. B, 1999, $30 \quad 60,11666-11677$.

111.F. Finger, J. Muller, C. Malten, R. Carius and H. Wagner, J. NonCryst. Solids, 2000, 266, 511-518.

112.W. Fuhs, J. Non-Cryst. Solids, 2008, 354, 2067-2078.

113.L. Korte, A. Laades and M. Schmidt, J. Non-Cryst. Solids, 2006, 352,

35 1217-1220.

114.D. L. Staebler and C. R. Wronski, Appl. Phys. Lett., 1977, 31, 292294.

115.M. Stutzmann and D. K. Biegelsen, Phys. Rev. B, 1989, 40, 98349840.

40 116.T. Umeda, S. Yamasaki, J. Isoya and K. Tanaka, Phys. Rev. B, 1999, 59, 4849-4857.

117.Y. Mai, S. Klein, R. Carius, H. Stiebig, X. Geng and F. Finger, Appl. Phys. Lett., 2005, 87, 073503.

118.M. Stutzmann and R. A. Street, Phys. Rev. Lett., 1985, 54, 1836$45 \quad 1839$.

119.S. Hasegawa, S. Narikawa and Y. Kurata, Philos. Mag. B, 1983, 48, 431-447.

120.F. Finger, J. Mueller, C. Malten and H. Wagner, Philos. Mag. B, 1998, 77, 805-830.

50 121.M. Kondo, T. Nishimiya, K. Saito and A. Matsuda, J. Non-Cryst. Solids, 1998, 230, 1031-1035.

122.P. Kanschat, K. Lips, R. Bruggemann, A. Hierzenberger, I. Sieber and W. Fuhs, Amorphous and Microcrystalline Silicon Technology-1998, 1999, 507, 793-798.

55 123.M. S. Brandt and M. Stutzmann, Phys. Rev. B, 1991, 43, 5184-5187.

124.K. Lips, S. Schuette and W. Fuhs, Philos. Mag. B, 1992, 65, 945-959.

125.M. Stutzmann, D. K. Biegelsen and R. A. Street, Phys. Rev. B, 1987, 35, 5666-5701.
126.A. Schweiger, C. Gemperle and R. R. Ernst, J. Magn. Reson., 1990,

$60 \quad \mathbf{8 6}, 70-81$.

127.L. Houben, M. Luysberg and R. Carius, Phys. Rev. B, $2003,67$.

128.T. Brammer, H. Stiebig and K. Lips, Appl. Phys. Lett., 2004, 85, 1625-1626.

129.T. Matsuyama, N. Terada, T. Baba, T. Sawada, S. Tsuge, K.

65 Wakisaka and S. Tsuda, J. Non-Cryst. Solids, 1996, 198, 940944.

130.R. B. Bergmann, G. Oswald, M. Albrecht and V. Gross, Sol. Energy Mat. Sol. Cells, 1997, 46, 147-155.

131.T. Sontheimer, C. Becker, U. Bloeck, S. Gall and B. Rech, Appl. $70 \quad$ Phys. Lett., 2009, 95, 101902.

132.M. A. Green, K. Emery, Y. Hishikawa, W. Warta and E. D. Dunlop, Prog. Photovol.: Res. Appl., 2011, 19, 565-572.

133.M. N. van den Donker, S. Klein, B. Rech, F. Finger, W. M. M. Kessels and M. C. M. van de Sanden, Appl. Phys. Lett., 2007,

90, 183504-183503.
134.N. H. Nickel, Appl. Phys. Lett., 1993, 62, 3285-3287.

135.T. Mishima, M. Taguchi, H. Sakata and E. Maruyama, Sol. Energy Mat. Sol. Cells, 2011, 95, 18-21.

136.V. A. Dediu, L. E. Hueso, I. Bergenti and C. Taliani, Nature Mater., $80 \quad 2009,8,707-716$.

137.L. S. Swanson, J. Shinar, A. R. Brown, D. D. C. Bradley, R. H. Friend, P. L. Burn, A. Kraft and A. B. Holmes, Synth. Met., 1993, 55, 241-248.

138.C. F. O. Graeff, M. S. Brandt, R. M. Faria and G. Leising, phys. stat. $85 \quad$ sol. (a), 1997, 162, 713-721.

139.T. Eickelkamp, S. Roth and M. Mehring, Mol. Phys., 1998, 95, $967-$ 972.

140.G. B. Silva, L. F. Santos, R. M. Faria and C. F. O. Graeff, Physica B, 2001, 308, 1078-1080.

90 141.D. R. McCamey, K. J. van Schooten, W. J. Baker, S. Y. Lee, S. Y. Paik, J. M. Lupton and C. Boehme, Phys. Rev. Lett., 2010, 104, 017601.

142.Y. Li, Y. Cao, J. Gao, D. Wang, G. Yu and A. J. Heeger, Synth. Met., 1999, 99, 243-248.

95 143.Y. X. Liu, M. A. Summers, S. R. Scully and M. D. McGehee, J. Appl. Phys., 2006, 99, 093521.

144.J. De Ceuster, E. Goovaerts, A. Bouwen, J. C. Hummelen and V. Dyakonov, Phys. Rev. B, 2001, 64, 195206.

145.A. Aguirre, P. Gast, S. Orlinskii, I. Akimoto, E. J. J. Groenen, H. El Mkami, E. Goovaerts and S. Van Doorslaer, Phys. Chem. Chem. Phys., 2008, 10, 7129-7138.

146.Y. N. Molin, O. A. Anisimov, V. M. Grigoryants, V. K. Molchanov and K. M. Salikhov, J. Phys. Chem., 1980, 84, 1853-1856.

147.Y. Araki, K. Maeda and H. Murai, Chem. Phys. Lett., 2000, 332, 515$105 \quad 520$.

148.V. R. Gorelik, K. Maeda, H. Yashiro and H. Murai, J. Phys. Chem. A, 2001, 105, 8011-8017.

149.N. S. Sariciftci, L. Smilowitz, A. J. Heeger and F. Wudl, Science, 1992, 258, 1474-1476.

110 150.T. P. Nguyen, P. Le Rendu, O. Gaudin, A. J. T. Lee, R. B. Jackman and C. H. Huang, Thin Solid Films, 2006, 511, 338-341.

151.A. Kadashchuk, V. I. Arkhipov, C. H. Kim, J. Shinar, D. W. Lee, Y. R. Hong, Y. R. Hong, P. Heremans and H. Baessler, Phys. Rev. B, 2007, 76, 235205.

115 152.D. P. McMahon and A. Troisi, Phys. Chem. Chem. Phys., 2011, 13, 10241-10248. 
153.A. J. Schellekens, W. Wagemans, S. P. Kersten, P. A. Bobbert and B. Koopmans, Phys. Rev. B, 2011, 84, 075204.

154.J. Lee, K. Vandewal, S. R. Yost, M. E. Bahlke, L. Goris, M. A. Baldo, J. V. Manca and T. Van Voorhis, J. A. Chem. Soc.,

$5 \quad$ 2010, 132, 11878-11880.

155.J. Kniepert, M. Schubert, J. C. Blakesley and D. Neher, J. Phys. Chem. Lett., 2011, 2, 700-705.

156.J. Behrends, A. Sperlich, A. Schnegg, T. Biskup, C. Teutloff, K. Lips, V. Dyakonov and R. Bittl, Phys. Rev. B, 2012, 85, 125206.

10

157.H. Morishita, L. S. Vlasenko, H. Tanaka, K. Semba, K. Sawano, Y. Shiraki, M. Eto and K. M. Itoh, Phys. Rev. B, 2009, 80.

158.A. Schnegg, A. A. Dubinskii, M. R. Fuchs, Y. A. Grishin, E. P. Kirilina, W. Lubitz, M. Plato, A. Savitsky and K. Mobius, Appl. Magn. Res., 2007, 31, 59-98.

15

159.C. J. Pickard and F. Mauri, Phys. Rev. Lett., 2002, 88, -.

160.U. Gerstmann, M. Rohrmuller, F. Mauri and W. G. Schmidt, phys. stat. sol. (c), 2010, 7, 157-160.

161.G. Pfanner, C. Freysoldt and J. Neugebauer, Phys. Rev. B, 2011, 83.

20 162.M. Fehr, A. Schnegg, B. Rech, K. Lips, O. Astakhov, F. Finger, G. Pfanner, C. Freysoldt, J. Neugebauer, R. Bittl and C. Teutloff, Phys. Rev. B, 2011, 84, 245203.

163.M. Kaupp, M. Bühl and V. G. Malkin, Calculation of NMR and EPR parameters : theory and applications, Wiley-VCH, Weinheim,

25 2004.

164.H. Moons, Ł. Łapok, A. Loas, S. Van Doorslaer and S. M. Gorun, Inorg. Chem., 2010, 49, 8779-8789. 